\title{
PERTURBATION OF TRANSLATION INVARIANT POSITIVITY PRESERVING SEMIGROUPS ON $L^{2}\left(\mathbf{R}^{N}\right)$ \\ BY
}

IRA W. HERBST AND ALAN D. SLOAN'

\begin{abstract}
ABstruct. The theory of singular local perturbations of translation invariant positivity preserving semigroups on $L^{2}\left(\mathbf{R}^{N}, d^{N} x\right)$ is developed. $A$ powerful approximation theorem is proved which allows the treatment of a very general class of singular perturbations. Estimates on the local singularities of the kernels of the semigroups, $e^{-t H}$, are given. Eigenfunction expansions are derived. The local singularities of the eigenfunction and generalized eigenfunctions are discussed. Results are illustrated with examples involving singular perturbations of $-\Delta$.
\end{abstract}

I. Introduction. The sum of an operator, $H_{0}$, which generates a positivity preserving translation invariant semigroup on $L^{2}\left(\mathbf{R}^{N}, d^{N} x\right)$ and a potential $V$ is the subject of the present work. In \$II the class of such $H_{0}$ 's is discussed more fully. Here we only remark that the operators corresponding to nonrelativistic and relativistic energy in quantum mechanics are included. The potentials considered are, in general, too singular to be operators and are given as forms, so that $H_{0}+V$ must be defined as a form sum. A detailed description of the potentials is given in 8III and IV. The success of the perturbation program for the investigation of operator sums is impressive [20]. For form sums such an analysis is more difficult because functions of forms are generally undefined.

One technique for analyzing functions, $f$, of $H_{0}+V$ is to:

(1) approximate $V$ by bounded functions $V_{n}$;

(2) show $f\left(H_{0}+V_{n}\right)$ approximates $f\left(H_{0}+V\right)$; and

(3) analyze $f\left(H_{0}+V\right)$ by (2) and a direct analysis of $f\left(H_{0}+V_{n}\right)$.

Such a procedure for (1) and (2) was developed by Kato [20] and Faris [10], using $f(x)=(x+\lambda)^{-1}$. It employs monotone convergence arguments and so is applicable only when the potential $V$ can be written as the sum of a rather general nonnegative function $V_{+}$and a nonpositive function $V_{-}$which is a small form perturbation of $H_{0}$. One truncates $V_{+}$and $V_{-}$to obtain functions $V_{+, n}$ and $V_{-, n}$ which are absolutely bounded by the integer $n$. Then, for all

Received by the editors August 16, 1976.

AMS (MOS) subject classifications (1970). Primary 47D05; Secondary $60 \mathrm{~J} 35$.

Key words and phrases. Positivity preserving, Levy-Khintchine formula, semigroups, singular perturbations, generalized eigenfunctions.

'Both authors were supported in part by NSF MPS 74-22844.

- American Mathematical Society 1978 
nonnegative $H_{0}$ one can show

$$
\begin{aligned}
& \underset{n \rightarrow \infty}{\text { strong limit strong limit }}\left(H_{0}+V_{+, n}+V_{-, m}+\lambda\right)^{-1} \\
& =\left(H_{0}+V+\lambda\right)^{-1}
\end{aligned}
$$

for all sufficiently large $\lambda$.

In contrast, we develop a technique to accommodate a potential $V$ which is the sum of a rather general nonnegative function $V_{+}$and a small form perturbation $V_{d}$ of $H_{0}$ which is given by a distribution $\tau$. That is, $V_{d}(f$, $g)=\tau(\bar{g})$ for all $f, g$ in $C_{c}^{\infty}$ so that $V_{d}$ need not be negative nor even a function. By regularizing $\tau$ we show the existence of $C_{c}^{\infty}$ functions $V_{n}$ such that

$$
\underset{n \rightarrow \infty}{\text { strong limit }}\left(H_{0}+V_{n}+\lambda\right)^{-1}=\left(H_{0}+V+\lambda\right)^{-1}
$$

for all sufficiently large $\lambda$. Note that (1.3) implies

$$
\underset{n \rightarrow \infty}{\text { strong limit }} e^{-t\left(H_{0}+V_{n}\right)}=e^{-t\left(H_{0}+V\right)}
$$

for all $t>0$.

The approximation theorem (see $\S \mathrm{V}$ ) is basic to our further developments. It allows the use of techniques of proof which only work for smooth perturbations so long as any estimates involved are uniform in the relevant parameters and as long as the result involved is stable under strong resolvent convergence. To our knowledge the approximation theorem is new even in the case $H_{0}=-\Delta$.

The approximation theorem is first used to show that $e^{-t H}$ is positivity preserving for $H=H_{0}+V$. In §VIII we derive an inequality which is useful in controlling the local singularities of the kernel of the integral operator $e^{-t H}$. We show this kernel is almost everywhere nonnegative and in $L P_{o c}\left(R^{N}\right.$ $\times \mathbf{R}^{N}$ ) for some $p>1$.

These results and other Sobolev type inequalities are used in §IX to prove local regularity theorems for the $L^{2}$ eigenfunctions of $H$. We illustrate this using $H_{0}=-\Delta$ and a potential behaving like $-\lambda^{2} / r^{2}$ at $r=0$.

In $\S \mathrm{X}$ we consider a subclass of the unperturbed operators introduced in §II. We show that if $\pm V_{d}<a H_{0}+b$ with $a<\frac{1}{2}$ and $V=V_{d}+V_{+}$, then $e^{-t\left(H_{0}+V\right)}$ is a Carleman operator. Consequently we obtain a generalized eigenfunction expansion for $H_{0}+V$. We analyze the local singularity structure of the generalized eigenfunctions by discussing their $L^{p}$ properties.

II. The unperturbed operators. Throughout this paper our unperturbed operators $H_{0}$ will be selfadjoint operators on $L^{2}\left(\mathbf{R}^{N}, d x\right), d x$ being Lebesgue measure on $\mathbf{R}^{N}$, and having the form 


$$
H_{0}=\mathscr{F} M_{h_{0}} \mathscr{F}^{-1}
$$

where $\mathscr{F}$ denotes the Fourier transform and $M_{h_{0}}$ denotes the selfadjoint operator consisting of multiplication by a real valued function $h_{0}$ satisfying the conditions:

$$
\mathrm{A}_{1}: h_{0}: \mathbf{R}^{N} \rightarrow[0, \infty) \text { is continuous and } h_{0}(0)=0 .
$$$$
\mathrm{A}_{2}: \text { For each } t>0, e^{-t h_{0}} \text { is in } L^{1}\left(\mathbf{R}^{N}, d x\right) \text {. }
$$

$A_{3}:$ For each $t>0, e^{-t h_{0}}$ is a positive definite function.

Such an $h_{0}$ will be called a function of type $A$ and the corresponding operator, $H_{0}$, will be called an operator of type $A$. The condition $h_{0}(0)=0$ has been chosen entirely for convenience. To evaluate the significance of these conditions we state the following lemma. Its conclusions are a consequence of Bochner's theorem and we omit the proof.

LEMMA 2.1. Let $H=\mathscr{F} M_{h} \mathscr{F}^{-1}$ be selfadjoint and bounded below on $L^{2}\left(R^{N}\right.$, $d x)$. Fix $t>0$. Then, $e^{-t H}$ is an integral operator with an a.e. nonnegative kernel in $L^{\infty}$ if and only if $e^{-t h}$ is in $L^{1}$ and $h=h^{*}$ a.e., where $h^{*}$ is continuous and $e^{-t h^{*}}$ is positive definite.

We remark that $A_{3}$ is a severe restriction on the class of functions $h_{0}$ which we will consider. However, the class is sufficiently rich to contain interesting examples, which we will shortly present. The following characterization, which is a specialization of the multi-dimensional version of the LevyKhintchine formula, can be useful. We set $v^{2}=|v|^{2}$ for $v$ in $\mathbf{R}^{N}$ where $|v|$ is the Euclidean norm of $v$.

LEMMA 2.2. The function $h_{0}$ satisfies $A_{1}$ and $A_{3}$ if and only if there exist a finite regular Borel measure $\mu$ on $\mathbf{R}^{N}$ and a real positive semidefinite matrix $C$ such that

$$
h_{0}(p)=\int_{|\lambda|>0} \lambda^{-2}\left(1+\lambda^{2}\right)(1-\cos (\lambda \cdot p)) d \mu(\lambda)+\sum_{i j} p_{i} C_{u} p_{j}
$$

This formula follows from Theorem 3, p. 188 of [13]. Note that, in particular, equation (2.3) implies $h_{0}(p)<\alpha\left(1+p^{2}\right)$ for some $\alpha>0$.

It is a familiar fact that second order elliptic differential operators with constant coefficients generate Brownian motion. Writing the Fourier transform of $1-\cos (\lambda \cdot p)$ as $\delta(x)-\frac{1}{2} \delta(x+\lambda)-\frac{1}{2} \delta(x-\lambda)$ shows that if $h_{0}$ is given by (2.3) then $H_{0}=\mathscr{F} M_{h_{0}} \mathscr{F}^{-1}$ is given by 


$$
\begin{aligned}
&\left(f, H_{0} g\right)= \sum_{i j} C_{i j}\left(\partial_{i} f, \partial_{j} g\right) \\
&+\frac{1}{2} \int(\bar{f}(x+\lambda)-\bar{f}(x))(g(x+\lambda)-g(x)) \\
& \cdot(1+\lambda)^{2} \lambda^{-2} d x d \mu(\lambda) .
\end{aligned}
$$

Thus, in some sense, $H_{0}$ generalizes the operator $-\Sigma_{i j} \partial_{i} C_{i j} \partial_{j}$. The representation of equation (2.3)' also leads to a simple proof that if $H_{0}$ is given by (2.3) then $e^{-t H_{0}}$ is positivity preserving [2] and [12].

The requirement that $h_{0}$ be nonnegative guarantees that $\left\{e^{-t H_{0}}\right\}_{1>0}$ is a contraction semigroup on $L^{2}$. We summarize some of the relevant properties of $e^{-t H_{0}}$ in the following theorem. Here, and in what follows, $\|T\|_{p, q}$ denotes the norm of an operator $T: L^{p} \rightarrow L^{q}$.

TheOREM 2.3. Assume $h_{0}$ is a function of type $A$. Then $e^{-t H_{0}}$ extends to a contraction semigroup on all the $L^{p}$ spaces for $p$ in $[1, \infty]$. On each of these spaces $e^{-t H_{0}}$ is, for $t>0$, a convolution operator:

$$
e^{-t H_{0}}=K_{t}^{0} * f
$$

with kernel $K_{t}^{0}$ in $L^{1} \cap L^{\infty}$ and $K_{t}^{0}$ nonnegative. $K_{t}^{0}$ is given by

$$
K_{t}^{0}=(2 \pi)^{-N / 2} \mathscr{F}\left(e^{-t h_{0}}\right) \text {. }
$$

In addition, for $t>0, e^{-t H_{0}}$ is a bounded map from $L^{1}$ to $L^{\infty}$ with

$$
\left\|e^{-t H_{0}}\right\|_{1, \infty}<(2 \pi)^{-N}\left\|e^{-t h_{0}}\right\|_{1} \text {. }
$$

The proof of Theorem 2.3 follows from [16], duality and interpolation.

Because it may be difficult in practice to determine whether a given function $h_{0}$ satisfies condition $A_{3}$ we now give some examples of type $A$ functions.

EXAMPLE 2.4. The nonrelativistic kinetic energy $h_{0}(p)=p^{2}$ is of type $A$. We have

$$
K_{t}^{0}(x)=(4 \pi t)^{-N / 2} e^{-x^{2} / 4 t}
$$

and $H_{0}=-\Delta$.

EXAMPLE 2.5. The relativistic kinetic energy $h_{0}(p)=\left(p^{2}+m^{2}\right)^{1 / 2}-m$, $m>0$, is of type $A$. Here ${ }^{2}$

$$
K_{t}^{0}(x)=(2 \pi)^{-N}\left(x^{2}+t^{2}\right)^{-1 / 2} t e^{m t} \int_{\mathbb{R}^{N}} e^{-\left(x^{2}+t^{2}\right)^{1 / 2}\left(p^{2}+m^{2}\right)^{1 / 2}} d p .
$$

EXAMPLE 2.6. A direct sum of relativistic and nonrelativistic kinetic energies

${ }^{2}$ One of the authors (I. H.) would like to thank L. J. Landau for a conversation during which equation (2.7) was derived. 


$$
h_{0}(p)=\sum_{i=1}^{J}\left(p_{i}^{2}+m_{i}^{2}\right)^{1 / 2}-m_{i}+\sum_{i=J+1}^{M} \frac{p_{i}^{2}}{2 m_{i}}
$$

is of type $A$, with $N=3 M$.

Given a function, $h_{0}$, of type $A$ it is easy to find others. Suppose $g$ is a continuous map from $[0, \infty)$ to $[0, \infty)$ satisfying

(a) $g(0)=0$;

(b) $g$ is $C^{\infty}$ away from 0 and $(-1)^{n} g^{(n+1)}(x) \geqslant 0$ for $x>0$; and

(c) $e^{-t\left(8 \cdot h_{0}\right)}$ is in $L^{1}$ for each $t>0$.

Then $g \circ h_{0}$ is also a function of type $A$. This follows from the theory of completely monotone functions [11]. Thus the fact that $h_{0}(p)=\left(p^{2}+m^{2}\right)^{1 / 2}$ - $m$ is of type $A$ follows from Example 2.4.

While we have assumed type $A$ functions to be continuous the additional type $A$ hypotheses imply that $\left(h_{0}+\lambda\right)^{1 / 2}$ is uniformly continuous for $\lambda>0$. In more detail we have

LeMmA 2.7. Suppose $h_{0}$ is given by equation (2.3). Let $\lambda>0$. Then for any $\varepsilon>0$ there is a $c(\varepsilon)<\infty$ such that for all $p, q$ in $\mathbf{R}^{n}$

$$
\left|\left(h_{0}(p)+\lambda\right)^{1 / 2}-\left(h_{0}(q)+\lambda\right)^{1 / 2}\right|<c(\varepsilon)|p-q|+\varepsilon .
$$

Proof. Choose $r$ large enough so that $2 \int_{|\lambda|>r}|\lambda|^{-2}\left(1+\lambda^{2}\right) d \mu(\lambda)<\varepsilon^{2} / 2$ and set $d \nu(\lambda)=\left(1+\lambda^{2}\right) x_{r}(\lambda) d \mu(\lambda)$ where $\chi_{r}(\lambda)=1$ if $0<|\lambda|<r$ and zero otherwise. Also set $q_{1}(p)=\int|\lambda|^{-2}(1-\cos (\lambda \cdot p)) d \nu(\lambda)$ and $q_{2}(p)=(p, C p)$. Note that

$$
\left|\nabla q_{2}(p)\right|^{2}=4|C p|^{2} \leqslant 4\|C\| q_{2}(p)
$$

Also,

$$
\begin{aligned}
\left|\left(\nabla_{i} q_{1}\right)(p)\right| & <\int\left|\lambda_{i} \sin (\lambda \cdot p)\right||\lambda|^{-2} d \nu(\lambda) \\
& <2 \int|\lambda|^{-1}\left|\lambda_{i}\right||\lambda|^{-1}\left|\sin \left(\frac{\lambda \cdot p}{2}\right)\right| d \nu(\lambda)
\end{aligned}
$$

so that

$$
\left|\nabla q_{1}(p)\right|^{2}<2 v\left(\mathbf{R}^{N}\right) \int 2|\lambda|^{-2} \sin ^{2}\left(\frac{\lambda \cdot p}{2}\right) d \nu(\lambda)=2 \nu\left(\mathbf{R}^{N}\right) q_{1}(p) .
$$

Let $q_{0}=q_{1}+q_{2}$ and $f=h_{0}-q_{0} \geqslant 0$. Note that $f<\varepsilon^{2} / 2$. Using Taylor's formula and the triangle inequality we find, for some $z$ between $p$ and $q$, that 


$$
\begin{aligned}
\mid\left(h_{0}(p)+\right. & \lambda)^{1 / 2}-\left(h_{0}(q)+\lambda\right)^{1 / 2} \mid \\
& <\left|\left(q_{0}(p)+\lambda+f(p)\right)^{1 / 2}-\left(q_{0}(q)+\lambda+f(p)\right)^{1 / 2}\right| \\
& +\left|\left(q_{0}(q)+\lambda+f(p)\right)^{1 / 2}-\left(q_{0}(q)+\lambda+f(q)\right)^{1 / 2}\right| \\
& <\frac{1}{2}|p-q|\left|\nabla q_{0}(z)\right|\left(\lambda+f(p)+q_{0}(z)\right)^{-1 / 2}+|f(p)-f(q)|^{1 / 2} \\
& <c(\varepsilon)|p-q|+\varepsilon .
\end{aligned}
$$

We will later make use of the following corollary:

COROLlary 2.8. Suppose $g$ is in the Schwartz space $S\left(\mathbf{R}^{N}\right)$ of rapidly decreasing functions on $\mathbf{R}^{N}$. For $\lambda>0$ define $Q_{\lambda}=\left(H_{0}+\lambda\right)^{1 / 2} M_{g}-M_{g}\left(H_{0}\right.$ $+\lambda)^{1 / 2}$. Then for every $\varepsilon>0$ there is a constant $b(\varepsilon)$ such that

$$
\left\|Q_{\lambda}\right\|<\int(b(\varepsilon)|p|+\varepsilon)|\hat{g}(p)| d p .
$$

Proor. $\mathscr{F} Q_{\lambda} \mathscr{F}^{-1}$ is an integral operator with kernel

$$
\hat{Q}_{\lambda}(p, q)=(2 \pi)^{-N}\left\{\left(h_{0}(p)+\lambda\right)^{1 / 2}-\left(h_{0}(q)+\lambda\right)^{1 / 2}\right\} \hat{g}(p-q) .
$$

Thus by Lemma 2.7, for each $\delta>0$

$$
\left|\hat{Q}_{\lambda}(p, q)\right|<(2 \pi)^{-N}\{c(\delta)|p-q|+\delta\}|\hat{g}(p-q)| .
$$

The corollary now follows from Young's inequality.

In what follows we will derive certain relationships involving operators of type $A$ by verifying the relationships on $C_{c}^{\infty}\left(R^{N}\right)$, the space of all infinitely differentiable functions of compact support in $\mathbf{R}^{N}$. Extending the relationships to the whole of the form domain of $H_{0}$ will follow from the fact that $C_{c}^{\infty}\left(\mathrm{R}^{N}\right)$ is a core for $\left(H_{0}+\lambda\right)^{1 / 2}$ for $\lambda>0$ and $H_{0}$ of type $A$.

III. The singular potentials. In this section we define the class of local perturbations which will be considered in later theorems. For details on the relevant theory of quadratic forms the reader is referred to [10] and [20]. We end the section with a partial characterization of this class (Proposition 3.4) which will prove useful in $8 \mathrm{~V}$.

In [10] we find

THEOREM 3.1. Let $S, U$ be bounded below selfadjoint operators with $Q(S) \cap$ $Q(U)$ dense. Let $W$ be a small form perturbation of $S$, let $u$ be the form associated to $U$ and let $V=u+W$. Then the form sum $S+V$ is bounded below and selfadjoint. Furthermore $S+V=(S+u)+W=(S+W)+u$ and $Q(S+V)=Q(S) \cap Q(U)$.

We next distinguish a class of perturbations in

Definition 3.2. Let $H_{0}$ be an operator of type $A$. Let $V$ be a nonnegative 
function. We also let $V$ denote the form associated with the selfadjoint operator consisting of multiplication by the function $V$ on $L^{2}\left(\mathbf{R}^{N}\right)$. Let $W$ be a Hermitian form which is a small form perturbation of $H_{0}$.

The Hermitian form $Z$ given as the sum of the Hermitian forms $V$ and $W$ is called an $H_{0}$-perturbation of type $B$ providing:

(i) $Q(V) \cap Q\left(H_{0}\right)$ is dense; and

(ii) $W$ agrees with a distribution on $C_{c}^{\infty}\left(\mathbf{R}^{N}\right)$, i.e., there is a distribution $\tau$ such that for all $f, g$ in $C_{c}^{\infty}\left(\mathbf{R}^{N}\right)$ we have

$$
W(f, g)=\tau(\bar{f} g)
$$

$V$ is called a positive part of $Z$ and $W$ is called a distributional part of $Z . \tau$ is the distribution associated with $W$.

Directly from Theorem 3.1 there follows

CoRollary 3.3. Let $Z$ be an $H_{0}$-perturbation of type $B$. Then $H_{0}+Z$ is selfadjoint and bounded below.

REMARK. Let $W$ be a small form perturbation of $H_{0}$. A necessary and sufficient condition that $W$ be the distributional part of an $H_{0}$-perturbation of type $B$ is that, for all $f, g, h$ in $C_{c}^{\infty}\left(\mathbf{R}^{N}\right)$,

$$
W(f, g h)=W(\bar{g} f, h) .
$$

Furthermore, the distribution associated with $W$ is tempered.

In the next result it will be shown that an associated distribution is locally of the form $\left(H_{0}+1\right)^{1 / 2} \psi$ for some $\psi$ in $L^{2}$. More precisely we have

Proposition 3.4. Let $\tau$ be the distributional part of an $H_{0}$-perturbation of type $B$. Then, for every bounded open subset, $\Lambda$, of $\mathbf{R}^{N}$ there is a function $\psi_{\Lambda}$ in $L^{2}\left(\mathbf{R}^{N}\right)$ such that

$$
\tau(g)=\left(\psi_{\Lambda},\left(H_{0}+1\right)^{1 / 2} g\right)
$$

for all $g$ in $C_{c}^{\infty}(\Lambda)$.

Proof. Let $\chi$ be in $C_{c}^{\infty}\left(\mathbf{R}^{N}\right)$ and equal to one on $\Lambda$. Since the norm \|\|$_{+1}$ associated with the inner product $(f, g)_{+1}=\left(f,\left(H_{0}+1\right) g\right)$ satisfies $\|f\|_{+1}>$ $\|f\|$ on $C_{c}^{\infty}\left(\mathbf{R}^{N}\right)$ we may view $\mathcal{K}_{+1}$, the Hilbert space obtained by closing $C_{c}^{\infty}\left(\mathbf{R}^{N}\right)$ in \|\|$_{+1}$, as a subset of $L^{2}\left(\mathbf{R}^{N}\right)$. If $\mathcal{X}_{-1}$, with norm \|\|$_{-1}$, is the dual of $\mathcal{K}_{+1}$ then we make the necessary identifications which permit the view $\mathcal{K}_{+1} \subset L^{2} \subset \mathcal{K}_{-1}$.

The distribution $\tau_{\Lambda}$ given as $\tau_{\Lambda}(f)=\tau(\chi f)$ satisfies, for some $0<d<\infty$, $\left|\tau_{\Lambda}(f)\right|<d\left\|x_{\Lambda}\right\|_{+1}\|f\|_{+1}$ for all $f$ in $C_{c}^{\infty}\left(\mathbf{R}^{N}\right)$. Thus $\tau_{\Lambda}$ extends to a continuous linear functional on $\mathcal{K}_{+1}$. By the Riesz representation theorem, there is an $h$ in $\mathcal{K}_{-1}$ such that $\tau_{\Lambda}(f)=(h, f)$ for all $f$ in $\mathcal{K}_{+1}$. Since 
$\left(H_{0}+1\right)^{-1 / 2}$ maps $\mathcal{K}_{-1}$ onto $L^{2}$ isometrically, the lemma follows with $\psi_{\Lambda}=\left(H_{0}+1\right)^{-1 / 2} h$.

IV. Examples of type $B$ perturbations.

EXAMPLE 4.1. Let $H_{0}$ be any operator of type $A$ on $L^{2}\left(\mathbf{R}^{N}\right)$. Let $V$ be a locally $L^{1}$ function on the complement of a closed set of measure zero. If $V$ is bounded below then $V$ is an $H_{0}$-perturbation of type $B$ [10].

EXAMPLE 4.2. Let $H_{0}=-\Delta$. Let $W$ be in $L^{p}+L^{\infty}$ for some $p$ satisfying $p>1$ if $N=1, p>1$ if $N=2$ and $p>N / 2$ if $N>3$. Then $W$ is the distributional part of an $H_{0}$-perturbation of type $B$ and 0 is the $H_{0}$ bound for $W[10]$.

EXAMPLE 4.3. Let $N=1$. Let the distribution associated with $W$ be the Fourier transform of an $L^{\infty}$ function. Let $H_{0}=-d^{2} / d x^{2}$. Then 0 is the $H_{0}$-bound for $W$ and $W$ is the distributional part of an $H_{0}$-perturbation of type $B$. In particular, $W$ may be given by a $\delta$-function concentrated at any point $p$ of $\mathbf{R}^{1}$ or by the principal value of $1 / x[10]$.

EXAMPLE 4.4. (SEE [6]). Let $H_{0}=-\Delta$ and $V=\left(V_{1}, \ldots, V_{n}\right)$ with each $V_{i}$ a real measurable function on $\mathbf{R}^{N}$. We assume $\sum_{i=1}^{N} V_{i}^{2}<\frac{1}{4} a^{2}\left(H_{0}+2 b\right)$ for some $a<1$ and $0<b<\infty$. Define

$$
W(f, g)=-\sum_{i=1}^{N} \int V_{i}(x) \frac{\partial(\bar{f} g)}{\partial x_{i}}(x) d x
$$

Then [0] $\pm W<a\left(H_{0}+b\right)$. To see this, merely observe that

$$
\begin{aligned}
|W(f, f)| & =|2 \operatorname{Re}(V f \cdot \nabla f)| \\
& <2\|V f\|_{2}\|\nabla f\|_{2} \leqslant a\left[\left(f,\left(H_{0}+2 b\right) f\right)\left(f, H_{0} f\right)\right]^{1 / 2}<a\left(f,\left(H_{0}+b\right) f\right) .
\end{aligned}
$$

Thus, $W$ is the distributional part of an $H_{0}$-perturbation of type $B$. In particular if $W$ is a distributional directional derivative of an $L^{\infty}$ function, then $W$ is a type $B$ perturbation of $H_{0}$ and zero is an $H_{0}$-bound for $W$. As a concrete example one may choose $W(x)=e^{x} \cos \left(e^{x}\right)$ with $N=1$.

EXAMPLE 4.5. Suppose $N>3$ and $H_{0}=-\Delta$. Let $Z=\sum_{i=1}^{M} \tau_{i}$ where $\tau_{i}$ is a distribution which after a linear inhomogeneous change of variable becomes a distribution $S_{i}$ on $\delta\left(R^{3}\right)$, form bounded relative to $-\Delta$ (on $R^{3}$ ) with form bound zero. Then $Z$ is the distributional part of an $H_{0}$-perturbation of type $B$. Also 0 is the $H_{0}$-bound for $Z$. In particular this is the case if $S_{i}$ is in $L^{2}\left(\mathbf{R}^{3}\right)+L^{\infty}\left(\mathbf{R}^{3}\right)[26]$.

EXAMPLe 4.6. Let $N>1$ and let $H_{0}=-\Delta$. Let $S \subset \mathbf{R}^{N}$ be a compact $C^{1}$ hypersurface, i.e. $S=F^{-1}(\{0\})$ where $F: \mathbf{R}^{N} \rightarrow \mathbf{R}^{1}$ is $C^{1}$ and $\nabla F(x) \neq 0$ for each $x$ in $S$. Let $\delta_{F}$ be the delta function concentrated on $S$ (as constructed in [29]. Then $\delta_{F}$ is associated with the distributional part of an $H_{0}$-perturbation 
of type $B$. Moreover, 0 is the $H_{0}$-bound for $\delta_{F}$. See Example 9.11 and [29] for more details.

V. The approximation theorem. The approximation theorem referred to in the introduction is the main result of this section, see Theorem 5.4. Throughout this section, $H_{0}$ will be an operator of type $A$. $\rho$ will be a $C_{c}^{\infty}\left(\mathbf{R}^{N}\right)$ function satisfying

(i) $\rho(x)=1$ whenever $\|x\|<1$; and

(ii) $0<\rho<1$.

We define $\beta_{n}(x)=\rho(x / n)$ and $\gamma_{n}(x)=\rho(n x)\left(\int \rho(n x) d x\right)^{-1}$.

If $T$ is an $H_{0}$-bounded Hermitian form then $T$ can be viewed as a bounded operator from the space $\mathcal{K}_{+1}$ normed with $\|f\|_{+1}=\left\|\left(H_{0}+1\right)^{1 / 2} f\right\|$ to the dual space $\mathcal{H}_{-1}$ normed with

$$
\|g\|_{-1}=\sup \left\{|(f, g)|:\|f\|_{+1}<1\right\} .
$$

Since $\left(H_{0}+\lambda\right)^{-1 / 2}: L^{2} \rightarrow \mathcal{H}_{+1}$ is bounded for all $\lambda>0$ we also have

$$
\left(H_{0}+\lambda\right)^{-1 / 2}: \mathcal{X}_{-1} \rightarrow L^{2}
$$

is bounded. Thus we may and will consider $\left(H_{0}+\lambda\right)^{-1 / 2} T\left(H_{0}+\lambda\right)^{-1 / 2}$ as a bounded linear map from $L^{2}$ to $L^{2}$.

We put $R_{0}^{1 / 2}(-\lambda)=\left(H_{0}+\lambda\right)^{-1 / 2}$.

LEMMA 5.1. Let $W$ be the distributional part of an $H_{0}$-perturbation of type $B$ with associated distribution $t$. Let $W_{n}(f, g)=t\left(\gamma_{n} *(\bar{f} g)\right)$, where * denotes convolution.

(a) If $\pm W<a H_{0}+b, a<1$, then $\pm W_{n}<a H_{0}+b$.

(b) If $\lambda>0$ then $R_{0}^{1 / 2}(-\lambda)\left(W_{n}-W\right) R_{0}^{1 / 2}(-\lambda)$ converges strongly to zero.

Proof. Let $f$ be in $C_{c}^{\infty}\left(\mathbf{R}^{N}\right)$. For $y$ in $\mathbf{R}^{N}$ define $f_{y}(x)=f(x-y)$. Because the Riemann sum approximations to the integral $\int \gamma_{n}(z)|f|^{2}(x-z) d z$ converge in the topology of $\delta$ we may interchange the action of $t$ and integration to get

$$
\pm W_{n}(f, f)= \pm t\left(\gamma_{n} *|f|^{2}\right)= \pm \int \gamma_{n}(y) t\left(\left|f_{y}\right|^{2}\right) d y .
$$

Thus $\pm W<a H_{0}+b$ implies

$$
\left|W_{n}(f, f)\right|<\int \gamma_{n}(y) d y\left(\left(a H_{0}+b\right) f, f\right) .
$$

Since $\int \gamma_{n}(y) d y=1$ part (a) of this lemma follows from the fact that $C_{c}^{\infty}$ is a core for $\left(H_{0}+1\right)^{1 / 2}$.

To prove part (b) suppose that $f$ and $g$ are in $C_{c}^{\infty}$ and choose $r$ so that $\rho(x)=0$ for $|x|>r$. Choose $\Lambda$ to be a bounded open set containing

$$
\left\{x \in \mathbf{R}^{N}: x=x_{1}+x_{2}, x_{1} \in \operatorname{supp}(g),\left|x_{2}\right|<r\right\} .
$$


We then have, using $\psi_{\Lambda}$ from Proposition 3.4,

$$
\begin{aligned}
W_{n}(f, g)-W & (f, g)=t\left(\gamma_{n} *(\bar{f} g)\right)-t(\bar{f} g) \\
= & \left(\psi_{\Lambda},\left(H_{0}+1\right)^{1 / 2}\left(\gamma_{n} * M_{g} \bar{f}-M_{g} \bar{f}\right)\right) \\
= & \left(\psi_{\Lambda},\left(\gamma_{n} *\left(H_{0}+1\right)^{1 / 2} M_{g}-\left(H_{0}+1\right)^{1 / 2} M_{g}\right) \bar{f}\right) \\
& =\left(\gamma_{n}^{\prime} * \psi_{\Lambda}-\psi_{\Lambda},\left(H_{0}+1\right)^{1 / 2} M_{g} \bar{f}\right)
\end{aligned}
$$

where $\gamma_{n}^{\prime}(x)=\gamma_{n}(-x)$. The third equality follows from the fact that convolution becomes multiplication under the Fourier transform.

Thus,

$$
\begin{aligned}
\left|W_{n}(f, g)-W(f, g)\right| & \\
& <\left\|\gamma_{n}^{\prime} * \psi_{\Lambda}-\psi_{\Lambda}\right\|\left\|\left(H_{0}+1\right)^{1 / 2} M_{z}\left(H_{0}+1\right)^{-1 / 2}\right\|\|f\|_{+1} .
\end{aligned}
$$

Setting $h=\left(H_{0}+\lambda\right)^{1 / 2} g$ and $w=\left(H_{0}+\lambda\right)^{1 / 2} f$ this inequality may be rewritten as

$$
\begin{aligned}
& \left|\left(w, R_{0}^{1 / 2}(-\lambda)\left(W_{n}-W\right) R_{0}^{1 / 2}(-\lambda) h\right)\right| \\
& \quad<c_{\lambda}\|w\|\left\|\gamma_{n}^{\prime} * \psi_{\Lambda}-\psi_{\Lambda}\right\|\left\|\left(H_{0}+1\right)^{1 / 2} M_{g}\left(H_{0}+1\right)^{-1 / 2}\right\| .
\end{aligned}
$$

From the facts that $\left\|\gamma_{n}^{\prime} * \psi_{\Lambda}-\psi_{\Lambda}\right\| \rightarrow 0$, that $\left\|\left(H_{0}+1\right)^{1 / 2} M_{g}\left(H_{0}+1\right)^{-1 / 2}\right\|$ $<\infty$ and that $C_{c}^{\infty}$ is a core for $\left(H_{0}+\lambda\right)^{1 / 2}$ we have $R_{0}^{1 / 2}(-\lambda)\left(W_{n}-\right.$ W) $R_{0}^{1 / 2}(-\lambda) h \rightarrow 0$ for all $h$ of the form $h=\left(H_{0}+\lambda\right)^{1 / 2} g, g$ in $C_{c}^{\infty}$. However, from part (a) of this lemma $\left\{R_{0}^{1 / 2}(-\lambda)\left(W_{n}-W\right) R_{0}^{1 / 2}(-\lambda): n=1,2, \ldots\right\}$ is uniformly bounded in operator norm. Consequently part (b) follows since $\left\{\left(H_{0}+\lambda\right)^{1 / 2} g: g \in C_{c}^{\infty}\right\}$ is dense.

LEMMA 5.2. Let $W$ and $t$ be as in Lemma 5.1. Define $W_{n}^{\prime}(f, g)=t\left(\beta_{n} \overline{\beta_{n}} g\right)$. Suppose $\pm W<a H_{0}+b, 0<a<1$ and $0<b<\infty$. Then

(a) $\pm W_{n}^{\prime}<\left(a+\varepsilon_{1}(n)\right) H_{0}+b+\varepsilon_{2}(n)$ with $\varepsilon_{i}(n) \rightarrow 0$ as $n \rightarrow \infty$ and $\varepsilon_{i}(n)$ dependent only on $a, b, \rho$ and $H_{0}$; and

(b) if $\lambda>0$ then $R_{0}^{1 / 2}(-\lambda)\left(W_{n}^{\prime}-W\right) R_{0}^{1 / 2}(-\lambda)$ converges strongly to zero and in fact for all $g$ in $L^{2}$,

$$
\begin{aligned}
& \left\|R_{0}^{1 / 2}(-\lambda)\left(W_{n}^{\prime}-W\right) R_{0}^{1 / 2}(-\lambda) g\right\| \\
& \quad<c_{\lambda}\left\{\left\|\left(M_{\beta_{n}}-1\right) g\right\|+\left\|\left(H_{0}+\lambda\right)^{1 / 2} M_{\beta_{n}}\left(H_{0}+\lambda\right)^{-1 / 2}-M_{\beta_{n}}\right\|\|g\|\right\} .
\end{aligned}
$$

Proof. Let

$$
\alpha_{n}=\left\|\left(a H_{0}+b\right)^{1 / 2} M_{\beta_{n}}-M_{\beta_{n}}\left(a H_{0}+b\right)^{1 / 2}\right\| .
$$

Corollary 2.8 implies that for each $\varepsilon>0$ 


$$
\alpha_{n}<\int(c(\varepsilon)|p|+\varepsilon)\left|\hat{\beta}_{n}(p)\right| d p=\int\left(\frac{c(\varepsilon)}{n}|p|+\varepsilon\right)|\hat{\rho}(p)| d p
$$

so that $\alpha_{n} \rightarrow 0$ as $n \rightarrow \infty$. Note however that

$$
\begin{aligned}
W_{n}^{\prime}(f, f) & <\left\|\left(a H_{0}+b\right)^{1 / 2} M_{\beta_{n}} f\right\|^{2} \\
& <\left(\alpha_{n}\|f\|+\left\|\left(a H_{0}+b\right)^{1 / 2} f\right\|\right)^{2} \\
& <\alpha_{n}^{2}\left(1+\delta^{-1}\right)\|f\|^{2}+(1+\delta)\left(f,\left(a H_{0}+b\right) f\right)
\end{aligned}
$$

for any $\delta>0$. Taking $\delta=\alpha_{n}$ completes the proof of part (a).

To prove (b) note that $W_{n}^{\prime}(f, g)-W(f, g)=t\left(\left(\beta_{n}+1\right) \bar{f}\left(\beta_{n}-1\right) g\right)$ so that

$$
\left|W_{n}^{\prime}(f, g)-W(f, g)\right|<c\left\|\left(M_{\beta_{n}}+1\right) f\right\|_{+1}\left\|\left(M_{\beta_{n}}-1\right) g\right\|_{+1} .
$$

If we note that

$$
\begin{aligned}
\|\left(M_{\beta_{n}}+1\right) & \left(H_{0}+\lambda\right)^{-1 / 2} f \|_{+1} \\
& <C_{\lambda}^{\prime}\left(1+\left\|\left(H_{0}+\lambda\right)^{1 / 2} M_{\beta_{n}}\left(H_{0}+\lambda\right)^{-1 / 2}\right\|\right)\|f\|<C_{\lambda}^{\prime \prime}\|f\|
\end{aligned}
$$

we then also see that

$$
\begin{aligned}
\sup _{\|\|_{2^{-1}}}\left|\left(\left(H_{0}+\lambda\right)^{-1 / 2} f,\left(W_{n}^{\prime}-W\right)\left(H_{0}+\lambda\right)^{-1 / 2} g\right)\right| \\
\quad<c_{\lambda}\left\{\left\|\left(M_{\beta_{n}}-1\right) g\right\|+\left\|\left(H_{0}+\lambda\right)^{1 / 2} M_{\beta_{n}}\left(H_{0}+\lambda\right)^{-1 / 2}-M_{\beta_{n}}\right\|\|g\|\right\}
\end{aligned}
$$

so that by Lemma 2.8

$$
\left(H_{0}+\lambda\right)^{-1 / 2}\left(W_{n}^{\prime}-W\right)\left(H_{0}+\lambda\right)^{-1 / 2} g \rightarrow 0 .
$$

This proves part (b).

For a preliminary version of the approximation theorem we consider only type $B$ perturbations with zero positive parts.

LemaA 5.3. Let $W, t$ be as in Lemma 5.1. There are $C_{c}^{\infty}$ functions, $w_{n}$, such that $\left\{\left(H_{0}+w_{n}\right)\right\}$ is uniformly bounded from below and such that $\left(H_{0}+w_{n}+\right.$ $\lambda)^{-1}$ converges strongly to $\left(H_{0}+W+\lambda\right)^{-1}$ for all sufficiently large positive $\lambda$.

Proof. Let $W_{n}(f, g)=t\left(\gamma_{n} * \bar{f} g\right)$ and $W_{n, m}(f, g)=t\left(\gamma_{n} *\left(\beta_{m} \bar{f} \beta_{m} g\right)\right)$. $W_{n}$ is integration against a $C^{\infty}$ function while $W_{n, m}$ is integration against a $C_{0}^{\infty}$ function, $w_{n, m}$; that is

$$
W_{n, m}(f, g)=\int_{\mathbf{R}^{N}} \bar{f}(x) w_{n, m}(x) g(x) d x .
$$

From Lemma 5.2, part (b), it follows that for any $g$ in $L^{2}$ 


$$
\begin{aligned}
& \left\|R_{0}^{1 / 2}(-\lambda)\left(W_{n, m}-W_{n}\right) R_{0}^{1 / 2}(-\lambda) g\right\| \\
& \quad<c_{\lambda}\left\{\left\|\left(M_{\beta_{m}}-1\right) g\right\|+\left\|\left(H_{0}+\lambda\right)^{1 / 2} M_{\beta_{m}}\left(H_{0}+\lambda\right)^{-1 / 2}-M_{\beta_{m}}\right\|\|g\|\right\}
\end{aligned}
$$

which is a bound independent of $n$. Thus

$$
\begin{aligned}
&\left\|R_{0}^{1 / 2}(-\lambda)\left(W-W_{n, m}\right) R_{0}^{1 / 2}(-\lambda) g\right\| \\
&<\left\|R_{0}^{1 / 2}(-\lambda)\left(W_{n, m}-W_{n}\right) R_{0}^{1 / 2}(-\lambda) g\right\| \\
&+\left\|R_{0}^{1 / 2}(-\lambda)\left(W-W_{n}\right) R_{0}^{1 / 2}(-\lambda) g\right\|
\end{aligned}
$$

which converges to zero as $n, m \rightarrow \infty$ by lemmas 5.1 and 5.2.

Define $A=R_{0}^{1 / 2}(-\lambda) W R_{0}^{1 / 2}(-\lambda)$ and $A_{n}=R_{0}^{1 / 2}(-\lambda) W_{n, n} R_{0}^{1 / 2}(-\lambda)$. From (a) of Lemmas 5.1 and 5.2 it follows that there is a $0<\eta<1$ such that if $\lambda>0$ is large enough then $\left\|A_{n}\right\|,\|A\|<\eta<1$ for all sufficiently large $n$. Finally observe that if $w_{n}=w_{n, n}$ then $\left\{H_{0}+w_{n}\right\}$ is uniformly bounded from below by (a) of Lemmas 5.1 and 5.2 while

$$
\begin{aligned}
\left(H_{0}+\right. & W+\lambda)^{-1}-\left(H_{0}+w_{n}+\lambda\right)^{-1} \\
& =\left(R_{0}^{1 / 2}(-\lambda)\right)\left((1+A)^{-1}-\left(1+A_{n}\right)^{-1}\right)\left(R_{0}^{1 / 2}(-\lambda)\right) \\
& =R_{0}^{1 / 2}(-\lambda)\left(\left(1+A_{n}\right)^{-1}\left(A_{n}-A\right)(1+A)^{-1}\right) R_{0}^{1 / 2}(-\lambda)
\end{aligned}
$$

converges strongly to zero since $\left\{\left\|\left(1+A_{n}\right)^{-1}\right\|: n=1,2, \ldots\right\}$ is uniformly bounded and since $A_{n}-A$ converges strongly to zero.

Approximation TheOREM 5.4. Let $Z$ be an $H_{0}$-perturbation of type $B$. There are $C_{c}^{\infty}$ functions $Z_{n}$ such that $\left(H_{0}+Z_{n}+\lambda\right)^{-1}$ converges strongly to $\left(H_{0}+Z\right.$ $+\lambda)^{-1}$ for all sufficiently large and positive $\lambda$.

Proof. Let $V$ be the positive part and $W$ be the distributional part of $Z$. Let $V_{n}(x)=V(x)$ if $0<V(x)<n$ and zero otherwise. That $\left(H_{0}+W+V_{n}\right.$ $+\lambda)^{-1}$ converges strongly to $\left(H_{0}+W+V+\lambda\right)^{-1}=\left(H_{0}+Z+\lambda\right)^{-1}$ follows from a monotone convergence argument [10].

Consider $V_{n}+W$ as the distributional part of an $H_{0}$-perturbation of type $B$. By the previous lemma there are $C_{c}^{\infty}$ functions, $z_{n, m}$ such that $B_{n, m}=\left(H_{0}\right.$ $\left.+z_{n, m}+\lambda\right)^{-1}$ converges strongly to $B_{n}=\left(H_{0}+V_{n}+W+\lambda\right)^{-1}$. Let $B=$ $\left(H_{0}+Z+\lambda\right)^{-1}$. Let $\left\{\phi_{i}\right\}_{i=1}^{\infty}$ be an orthonormal basis of $L^{2}\left(\mathbf{R}^{N}\right)$. Fix $n$. Choose $m_{1}(n)$ so that if $m \geqslant m_{1}(n)$ then $\left\|\left(B_{n, m}-B_{n}\right) \phi_{1}\right\|<1 / n$. Inductively, suppose $m_{1}(n)<m_{2}(n)<\cdots<m_{j-1}(n)$ have been chosen so that if $m>$ $m_{j-1}(n)$ then $\left\|\left(B_{n, m}-B_{n}\right) \phi_{i}\right\|<1 / n$ for $i=1,2, \ldots, j-1$. Then choose $m_{j}(n)>m_{j-1}(n)$ so that if $m>m_{j}(n)$ then

$$
\left\|\left(B_{n, m}-B_{n}\right) \phi_{j}\right\|<1 / n \text {. }
$$

Thus, if $j<n$ we have $m_{n}(n) \geqslant m_{j}(n)$ so that $\left\|\left(B_{n, m_{n}(n)}-B_{n}\right) \phi_{j}\right\|<1 / n$. 
Set $Z_{n}=z_{n, m_{n}(n)}$. Then for each $j$

$$
\begin{aligned}
& \left\|\left(\left(H_{0}+Z_{n}+\lambda\right)^{-1}-\left(H_{0}+Z+\lambda\right)^{-1}\right) \phi_{j}\right\| \\
& \quad<1 / n+\left\|\left(\left(H_{0}+V_{n}+W+\lambda\right)^{-1}-\left(H_{0}+Z+\lambda\right)^{-1}\right) \phi_{j}\right\|
\end{aligned}
$$

for all $n>j$. That is, $\left(H_{0}+Z_{n}+\lambda\right)^{-1}$ converges strongly to $\left(H_{0}+Z+\right.$ $\lambda)^{-1}$ on a dense set. Since $\left\{\left(H_{0}+Z_{n}+\lambda\right)^{-1}\right\}$ is uniformly bounded we conclude that $\left(H_{0}+Z_{n}+\lambda\right)^{-1}$ converges strongly to $\left(H_{0}+Z+\lambda\right)^{-1}$.

COROLlary 5.5. Let $Z$ be an $H_{0}$-perturbation of type $B$. There are $C_{c}^{\infty}$ functions, $Z_{n}$, such that $\left\{H_{0}+Z_{n}\right\}$ is uniformly bounded from below and such that $e^{-t\left(H_{0}+Z_{n}\right)}$ converges strongly to $e^{-t\left(H_{0}+Z\right)}$.

Proof. The previous theorem and a theorem of Kato [20] yield the result.

VI. Positivity preserving semigroups. An operator $T$ on $L^{2}$ is positivity preserving if $T(f)>0$ almost everywhere whenever $f>0$ almost everywhere.

Example 6.1. Let $H_{0}$ be an operator of type $A$. Then $e^{-t H_{0}}$ is positivity preserving for all $t>0$. This follows from Lemma 2.1.

TheOREM 6.2. Let $H_{0}$ be an operator of type $A$. Let $Z$ be an $H_{0}$-perturbation of type $B$. Then $e^{-t\left(H_{0}+Z\right)}$ is positivity perserving for all $t>0$.

Proor. First observe that strong limits of positivity preserving operators are positivity preserving. The Trotter product formula [36] shows that $e^{-t\left(H_{0}+Z\right)}$ is positivity preserving in case $Z$ is a real $L^{\infty}$ function. The corollary to the approximation theorem completes the proof.

COROLlary 6.3. Let $H_{0}$ be an operator of type $A$. Let $Z$ be an $H_{0^{-}}$ perturbation of type $B$. Then for all sufficiently large and positive $\lambda,\left(H_{0}+Z+\right.$ $\lambda)^{-1}$ is positivity preserving.

Proof. This corollary follows from the previous theorem upon writing the resolvent as a Laplace transform in terms of the semigroup [20].

VII. Weak limits of integral operators. This section contains two technical lemmas which not only permit the conclusion that certain weak limits of integral operators are integral operators but also preserve certain pointwise inequalities for kernels under weak limits. The lemmas will be used in the following section.

Notation. (a) If $1<p<\infty$ then $p^{\prime}$ denotes the number satisfying $p^{-1}+$ $\left(p^{\prime}\right)^{-1}=1$. Also $\infty^{\prime}=1$ and $l^{\prime}=\infty$.

(b) If $Q$ is any measurable subset of $\mathbf{R}^{N}$ and if $f$ is a measurable function defined on $Q$ then for $1<q<\infty$ the local $L^{q}$ norms are defined by 


$$
\begin{aligned}
C_{Q, q}(f) & =\left(\int_{Q}|f(x)|^{q} d x\right)^{1 / q}, \quad 1<q<\infty, \\
C_{Q, \infty}(f) & =\underset{x \in Q}{\operatorname{ess}-\sup }|f(x)| .
\end{aligned}
$$

Here $d x$ denotes Lebesgue measure as will $d y, d p$, and $d k$.

(c) By a partition of $\mathbf{R}^{N}$ we mean a countable collection of mutually disjoint subsets of finite Lebesgue measure having union $\mathbf{R}^{N}$.

LEMMA 7.1. Let $\left\{T_{k}\right\}$ be a sequence of bounded linear integral operators on $L^{2}\left(\mathbf{R}^{N}\right)$ which converge weakly to the bounded linear operator $T$. Let the kernels, $S_{k}$, of $T_{k}$ be almost everywhere nonnegative. Suppose there is a partition, $\mathfrak{N}$, of $\mathbf{R}^{N}$ and $a 1<p<\infty$ such that

$$
\sup _{k} C_{Q p}\left(S_{k}\right)<\infty
$$

for each $Q$ in $\mathfrak{N} \times \mathfrak{N}$.

Then $T$ is an integral operator with an almost everywhere nonnegative kernel $S$ satisfying

$$
C_{Q p}(S)<\sup _{k} C_{Q p}\left(S_{k}\right)
$$

and

$$
\lim _{k \rightarrow \infty} \int_{Q} f S_{k} d x=\int_{Q} f S d x
$$

for all $f$ in $L^{p^{\prime}}(Q, d x)$ and $Q$ in $\Re \times \Re$.

Proof. For each $Q=M_{2} \times M_{1}$ in $\Re \times \Re$ define a linear functional $l_{Q}$ on the dense vector subspace of $L^{p^{\prime}}(Q)$ consisting of all finite sums of the form

$$
\sum_{i=1}^{n} g_{i 2} \otimes g_{i 1}
$$

where $g_{i j} \in L^{\infty}\left(M_{j}\right)$, by the formula

$$
l_{Q}(f)=\lim _{k \rightarrow \infty} \sum_{i=1}^{n}\left(\bar{g}_{i 2}, T_{k}\left(g_{i 1}\right)\right) .
$$

$l_{Q}$ is well defined since it is also given by the formula

$$
l_{Q}(f)=\lim _{k \rightarrow \infty} \int_{Q} f d v_{k}
$$

where $d v_{k}(x, y)=S_{k}(x, y) d x d y$.

Since $\sup _{k} C_{Q_{p}}\left(S_{k}\right)=C_{Q}<\infty$ it follows from equation (7.5) that for $f$ of the above form

$$
\left|l_{Q}(f)\right|<C_{Q}\|f\|_{p^{\prime \prime}}
$$


Consequently, $l_{Q}$ extends uniquely to a bounded linear functional on $L^{P^{\prime}}(Q)$ and is therefore given by integration against some $L^{p}(Q)$ function, $S_{Q}$. Let $d v_{Q}=S_{Q} d x d y$. Since the above class of functions is dense in $L^{P^{\prime}}(Q)$ it follows from (7.5) that

$$
\lim _{k \rightarrow \infty} \int f d v_{k}=\int f d v_{Q}
$$

for all $f$ in $L^{p^{\prime}}(Q)$. Consequently

$$
S_{Q}>0 \text { a.e. }
$$

If $g_{j}$ is a nonnegative function in $L^{2}\left(M_{j}\right)$, let $\left\{g_{n j}\right\}$ be a sequence of nonnegative $L^{\infty}\left(M_{j}\right)$ functions which increase to $g_{j}$. Then by dominated convergence, $g_{n j}$ converges to $g_{j}$ in $L^{2}\left(M_{j}\right)$ so that

$$
\lim _{n \rightarrow \infty}\left(g_{n 2}, T\left(g_{n 1}\right)\right)=\left(g_{2}, T\left(g_{1}\right)\right)
$$

while by monotone convergence

$$
\lim _{n \rightarrow \infty} \int g_{n 2} \otimes g_{n 1} d v_{Q}=\int g_{2} \otimes g_{1} d v_{Q}
$$

Consequently,

$$
\left(g_{2}, T g_{1}\right)=\int \bar{g}_{2} \otimes g_{1} d v_{Q}
$$

for all nonnegative $g_{j}$ in $L^{2}\left(M_{j}\right)$.

Since each point of $\mathbf{R}^{2 N}$ is in exactly one $Q$ of $\Re \times \Re$, a measurable function $S$ may be defined by

$$
S(x, y)=S_{Q}(x, y) \quad \text { if }(x, y) \in Q .
$$

Let $g_{j}$ in $L^{2}\left(\mathbf{R}^{N}\right)$ be nonnegative. Then

$$
\text { (7.7) }\left(g_{2}, T g_{1}\right)=\sum_{Q \in \mathscr{R} \times \mathscr{R}} \int \bar{g}_{2} \otimes g_{1} S_{Q} d x d y=\int \bar{g}_{2} \otimes g_{1} S d x d y
$$

using a monotone convergence argument. Since any $L^{2}$ function may be written as a complex combination of four nonnegative $L^{2}$ functions the equalities of (7.7) are true for all $g_{j}$ in $L^{2}\left(\mathbf{R}^{N}\right)$ and so express $T$ as an integral operator. The kernel of $T, S$, is nonnegative a.e. by (7.6).

Remarks. (a) Strong (and so weak) limits of integral operators are, in general, not integral operators.

(b) It does not follow that the kernels of $T_{k}$ converge a.e. to the kernel of $T$.

Nevertheless, the convergence of the linear functionals, obtained as integration against the kernels locally, may be used to preserve pointwise inequalities for kernels. This has already been demonstrated for nonnegativity of kernels. The next lemma gives another inequality which is preserved and which will be useful in what follows. 
Notation. If $T$ is an integral operator we may write $T(\cdot, \cdot)$ for its kernel.

LEMMA 7.2. Let $\left\{S_{k}\right\}$ and $\left\{R_{k}\right\}$ be sequences of bounded linear operators on $L^{2}\left(R^{N}\right)$ converging weakly to the bounded linear operators $S$ and $R$, respectively. Suppose the hypotheses of Lemma 7.1 are satisfied for both sequences, with identical $p$ 's and $\Re$ 's. Let $G$ be an a.e. nonnegative function in $L^{\infty}(Q)$ for each $Q$ in $\mathfrak{N} \times \mathfrak{\pi}$. Fix $1<q<\infty$. If

$$
S_{k}(x, y)<G(x, y)\left(R_{k}(x, y)\right)^{1 / q} \text { a.e. }
$$

for each $k$ then

$$
S(x, y)<G(x, y)(R(x, y))^{1 / q} \text { a.e. }
$$

ProOF. Taylor's theorem implies that for every $\varepsilon>0$

$$
\begin{aligned}
\left(R_{k}(x, y)+\varepsilon\right)^{1 / q} & -(R(x, y)+\varepsilon)^{1 / q} \\
& <\left(R_{k}(x, y)-R(x, y)\right)(1 / q)(R(x, y)+\varepsilon)^{1 / q-1} .
\end{aligned}
$$

Consequently, for any $Q$ in $\Re \times \mathfrak{N}$ and any nonnegative $f$ in $L^{\infty}(Q)$ we find

$$
\underset{k}{\limsup } \int_{Q} G f\left(\left(R_{k}+\varepsilon\right)^{1 / q}-(R+\varepsilon)^{1 / q}\right)<\limsup _{k} \int_{Q} L\left(R_{k}-R\right)=0
$$

by Lemma 7.1 since

$$
L(x, y)=(1 / q) G(x, y) f(x, y)(R(x, y)+\varepsilon)^{1 / q-1}
$$

is in $L^{\infty}(Q)$ and so in $L^{p^{\prime}}(Q)$. But then

$$
\begin{aligned}
\int_{Q} S f & =\lim _{k \rightarrow \infty} \int_{Q} S_{k} f<\limsup \int_{Q} G f\left(R_{k}\right)^{1 / q} \\
& <\int_{Q} f G(R+\varepsilon)^{1 / q}+\limsup _{k} \int_{Q} G f\left(\left(R_{k}+\varepsilon\right)^{1 / q}-(R+\varepsilon)^{1 / q}\right) \\
& <\int_{Q} f G R^{1 / q}+\varepsilon^{1 / q} \cdot\|G\|_{\infty} \cdot \operatorname{measure}(Q) .
\end{aligned}
$$

Since $\varepsilon>0$ is arbitrary we find that for all nonnegative $f$ in $L^{\infty}(Q)$

$$
\int_{Q} S f \leqslant \int_{Q} G R^{1 / q_{f}}
$$

The lemma thus follows.

Remark. The hypothesis of identical p's and $\Re$ 's in the previous lemma was made only for convenience. If in fact the $p$ 's are different choose a new $q$ equal to the minimum of these and if the partitions are different let $T$ th be a partition which is a refinement of both partitions. Then the proof proceeds as before. 
VIII. Kernels of semigroups. As a result of the approximation theorem and Lemma 7.2 we are able to show that the semigroup, generated by $H_{0}+p Z$ for all $H_{0}$-perturbations of type $B, Z$, and all $p>1$ sufficiently small, consists of integral operators with a.e. nonnegative kernels. In addition, if $K_{p}$ denotes the kernel of $e^{-t H_{p}}$, for some fixed $t>0$, then

$$
K_{1} \leqslant\left(K_{0}\right)^{1 / p^{\prime}}\left(K_{p}\right)^{1 / p} \text { a.e. }
$$

for all sufficiently small $p>1$. This inequality (see [7] and the remarks following Lemma 8.1) will be used in the last two sections.

Notation. If $V$ is a real measurable function on $\mathbf{R}^{N}$ then multiplication by $V$ is a selfadjoint operator on $L^{2}\left(\mathbf{R}^{N}\right)$. We let $V$ additionally denote this selfadjoint operator and also its corresponding quadratic form.

LEMMA 8.1. Let $H_{0}$ be an operator of type $A$. Let $V$ be a real function in $L^{\infty}\left(\mathbf{R}^{N}\right)$. Then the operator sum, $H_{p}$, of $H_{0}$ and $p V$ is, for every $0<p<\infty$, selfadjoint and bounded below. For $t>0, e^{-t H}$, is an integral operator with $a$ kernel which satisfies

$$
0<e^{-t H_{1}}(x, y)<\left(e^{-t H_{0}}(x, y)\right)^{1 / p^{\prime}}\left(e^{-t H_{p}}(x, y)\right)^{1 / p}
$$

for almost every $(x, y)$ in $\mathbf{R}^{2 N}$ and every $1<p<\infty$.

Proof. That $H_{p}$ is selfadjoint and bounded below is well known [20]. The Trotter product formula [36]:

$$
e^{-t H_{q}}=\underset{n \rightarrow \infty}{\text { strong limit }} T_{n}^{q V}
$$

where $T_{n}^{q V}=\left(e^{-t H_{0} / n_{e}} e^{-t q V / n}\right)^{n}$, and the observation that $T_{n}^{q V}$ is explicitly an integral operator with kernel satisfying

$$
0 \leqslant T_{n}^{q V}(x, y) \leqslant e^{q t\|V\|_{\infty}} e^{-t H_{0}}(x, y)
$$

show the hypotheses of Lemma 7.1 are satisfied with any partition $\Re$ and any $1<p \leqslant \infty$ because $e^{-t H_{0}}(\cdot, \cdot) \in L^{\infty}$. Consequently, $e^{-t H_{p}}$ is an integral operator with an a.e. nonnegative kernel. Further, $e^{-t H_{p}}(\cdot, \cdot)$ is in $L^{\infty}\left(\mathbf{R}^{2 N}\right)$ because

$$
C_{Q, \infty}\left(e^{-t H_{\rho}}\right) \leqslant e^{t p\|V\|_{\infty}} C_{Q, \infty}\left(e^{-t H_{0}}\right)<e^{t p\|V\|_{\infty}\left\|e^{-t H_{0}}\right\|_{\infty} .}
$$

$$
\text { Let } K_{n}(x, y)=e^{-t H_{0} / n}(x, y) \text { and let } L_{n}(x)=e^{-t V(x) / n}=e^{-t V / n}(x) \text {. Then }
$$

$$
\begin{aligned}
K_{n}\left(x, z_{1}\right) & L_{n}\left(z_{1}\right) K_{n}\left(z_{1}, z_{2}\right) L_{n}\left(z_{2}\right) \cdots K_{n}\left(z_{n-1}, y\right) L_{n}(y) \\
= & \left(K_{n}\left(x, z_{1}\right) K_{n}\left(z_{1}, z_{2}\right) \cdots K_{n}\left(z_{n-1}, y\right)\right)^{1 / p^{\prime}} \\
& \cdot\left(K_{n}\left(x, z_{1}\right) L_{n / p}\left(z_{1}\right) \cdots K_{n}\left(z_{n-1}, y\right) L_{n / p}(y)\right)^{1 / p} .
\end{aligned}
$$

Thus, the semigroup property and Holder's inequality show

$$
0<T_{n}^{V}(x, y)<\left(e^{-t H_{0}}(x, y)\right)^{1 / p^{\prime}}\left(T_{n}^{p V}(x, y)\right)^{1 / p} .
$$


The lemma now follows from the Trotter product formula and Lemma 7.2.

RemarKs. (a) Similar techniques show that if $W, V$ are real functions in $L^{\infty}\left(R^{N}\right)$ then, almost everywhere,

$$
e^{-t\left(H_{0}+W+V\right)}(x, y)<\left(e^{-t\left(H_{0}+p^{\prime} W\right)}(x, y)\right)^{1 / p^{\prime}}\left(e^{-t\left(H_{0}+p V\right)}(x, y)\right)^{1 / p} .
$$

(b) If one begins with the Feynman-Kacs formula for $H_{0}=-\Delta$ :

$$
e^{-t\left(H_{0}+V\right)}(x, y)=\int e^{-\int_{0}^{\prime} V(w(s)) d s} d \mu_{x y}(w)
$$

where $\mu_{x y}$ denotes conditional Wiener measure, then the inequality follows directly from Hölder's inequality. Using this intuition, Deift and Simon [7] independently proved a similar inequality for $H_{0}=-\Delta$.

THEOREM 8.2. Let $H_{0}$ be an operator of type A. Let $Z$ be an $H_{0}$-perturbation of type B. Suppose the distributional part, $W$, of $Z$ satisfies

$$
\pm W<a H_{0}+b \text { for } 0<a<1, \infty>b>0 \text {. }
$$

Then, for $1<p<1 / a, e^{-t\left(H_{0}+p Z\right)}$ is an integral operator satisfying

$$
0<e^{-t\left(H_{0}+z\right)}(x, y)<\left(e^{-t H_{0}}(x, y)\right)^{1 / p^{\prime}}\left(e^{-t\left(H_{0}+p z\right)}(x, y)\right)^{1 / p}
$$

almost everywhere.

Proof. It is clear from Corollary 5.5 and from the proof of Theorem 5.4 that we can choose $Z_{n} \in C_{c}^{\infty}$ so that $e^{-t\left(H_{0}+r Z_{n}\right)}$ converges strongly to $e^{-t\left(H_{0}+r Z\right)}$ for all $0<r<p$.

Let $Q=Q_{1} \times Q_{2}$ where each $Q_{i}$ is a measurable subset of $\mathbf{R}^{N}$ with finite measure. Let $\chi_{Q}, \chi_{Q_{1}}, \chi_{Q_{2}}$ be the characteristic functions of $Q, Q_{1}, Q_{2}$, respectively. Fix $t>0$. Denote $e^{-t\left(H_{0}+r Z\right)}$ by $E_{r}$ and $e^{-t\left(H_{0}+r Z_{n}\right)}$ by $E_{r, n}$. Let $1<\alpha<1 / a$ and choose $\beta>1$ so that $1<q=\alpha \beta<1 / a$. Let $f \in L^{\beta^{\prime}}(Q)$.

By Lemma 8.1, $E_{\alpha, n}$ is an integral operator with an a.e. nonnegative kernel satisfying

$$
\begin{aligned}
& \left|\int_{Q} f(x, y) E_{\alpha, n}(x, y) d x d y\right| \\
& \quad<\int_{Q}|f(x, y)|\left(E_{0}(x, y)\right)^{1 / \beta^{\prime}}\left(E_{q, n}(x, y)\right)^{1 / \beta} d x d y \\
& \quad<\left(\int_{Q}|f(x, y)|^{\beta^{\prime}} E_{0}(x, y) d x d y\right)^{1 / \beta^{\prime}}\left(\int x_{Q}(x, y) E_{q, n}(x, y) d x d y\right)^{1 / \beta} \\
& \quad<\left\|e^{-t H_{0}}(\cdot, \cdot)\right\|_{\infty}^{1 / \beta^{\prime}} C_{Q, \beta^{\prime}}(f)\left(\chi_{Q_{1}}, E_{q, n} \chi_{Q_{2}}\right)^{1 / \beta} .
\end{aligned}
$$

Since $E_{q, n}$ converges strongly to $E_{q}$ we find that, after choosing $\beta$ in $(1,1 / p a)$,

$$
\sup _{n} C_{Q, \beta}\left(E_{1, n}\right)<\infty
$$

and 


$$
\sup _{n} C_{Q, \beta}\left(E_{p, n}\right)<\infty .
$$

Lemmas 7.1, 7.2, 8.1 and Corollary 5.5 complete the proof of the theorem. Using the Laplace transform formula for the resolvent, Theorem 8.2 leads to

COROLLARY 8.3. Under the hypotheses of the previous theorem, $\left(H_{0}+p Z+\right.$ $\lambda)^{-1}$ is, for all $\lambda \geqslant 0$ sufficiently large, an integral operator with an a.e. nonnegative kernel satisfying

$$
\left(H_{0}+Z+\lambda\right)^{-1}(x, y)<\left(\left(H_{0}+\lambda\right)^{-1}(x, y)\right)^{1 / p^{\prime}}\left(\left(H_{0}+p Z+\lambda\right)^{-1}(x, y)\right)^{1 / p}
$$

almost everywhere.

COROLlaRY 8.4. Under the hypotheses of the previous theorem the kernel of $e^{-t\left(H_{0}+Z\right)}$ is in $L^{\beta}(Q)$ for every bounded measurable subset $Q$ of $\mathbf{R}^{2 N}$ for $1<\beta<1 / a$.

Proof. This follows from (8.2) and (7.3).

IX. Regularity properties of eigenfunctions. It is well known that singularities of the potential, $V$, can cause singularities in the eigenfunctions of $-\Delta+V$. We consider to what extent these singularities are absent under suitable conditions on the perturbations. We consider all unperturbed $H_{0}$ of type $A$, but we later impose further restrictions on these unperturbed operators to extend our results.

For most of the purposes of this section, an eigenfunction is considered to be free of singularities if it belongs to $L^{\infty}$. However in Theorem 9.7 we isolate a class of $H=H_{0}+W$ for which the eigenfunctions are Hölder continuous of some order.

THEOREM 9.1. Let $H_{0}$ be an operator of type $A$ and suppose $\lambda_{0} Z$ is an $H_{0}$-perturbation of type $B$ for some $\lambda_{0}>1$. Then for all $t>0, e^{-t\left(H_{0}+Z\right)}$ extends to a bounded map of $L^{q}$ into $L^{r}$ for $q \leqslant r$ and $q, r$ in $\left[\left(2 \lambda_{0}\right)^{\prime},\left(2 \lambda_{0}\right)\right]$ where $\left(2 \lambda_{0}\right)^{\prime}=\left(1-\left(2 \lambda_{0}\right)^{-1}\right)^{-1}$.

Proof. Define $H_{\lambda}=H_{0}+\lambda Z$ for $\lambda$ in $\left[1, \lambda_{0}\right]$.

Suppose $q$ is in $\left[2,2 \lambda_{0}\right]$ and $p$ is in $\left[q / 2, \lambda_{0}\right]$. Then define $s$ in $[0,1]$ by the equation

$$
s / p^{\prime}+q / 2 p=1 \text {. }
$$

If $f$ is a nonnegative function in $L^{q}$ we have by Theorem 8.2 that

$$
\int e^{-t H}(x, y) f(y) d y<\int\left(e^{-t H_{0}}(x, y) f(y)^{s}\right)^{1 / p}\left(e^{-t H_{p}}(x, y) f(y)^{q / 2}\right)^{1 / p} d y .
$$

By Hölder's inequality we find, for almost every $x$, 


$$
\left(e^{-t H_{f}}\right)(x)<\left(e^{-t H_{0} s}\right)^{1 / p^{\prime}}(x)\left(e^{-t H_{0} f q / 2}\right)^{1 / p}(x) \text {. }
$$

Thus

$$
\left\|e^{-t H_{f}}\right\|_{2 p}<\left\|e^{-t H_{0}}\right\|_{q / s, \infty}^{1 / p^{\prime}}\left\|e^{-t H_{p}}\right\|_{2,2}^{1 / p}\|f\|_{q^{\circ}}
$$

By Theorem 2.3 and The Riesz-Thorin interpolation theorem, $\left\|e^{-t H_{0}}\right\|_{r, \infty}<$ $c(t)<\infty$ for all $r$ in $[1, \infty]$. Hence $(9.2)$ implies

$$
\left\|e^{-t H}\right\|_{q, p}<\infty
$$

if $q \in\left[2,2 \lambda_{0}\right]$ and $p \in\left[q, 2 \lambda_{0}\right]$.

The rest of the theorem is proved using duality and the semigroup property.

COROLLARY 9.2. Under the hypotheses of Theorem 9.1 all $L^{2}$ eigenfunctions of $H_{0}+Z$ are in $L^{p}$ for $p$ in $\left[2,2 \lambda_{0}\right]$.

REMARK. In cases where $h_{0}(p)$ grows like a power of $|p|$ at $\infty$, Corollary 9.2 will not be optimal. This is because all eigenfunctions of $H$ are in the domain of $\left(H_{0}+1\right)^{1 / 2}$ and $\left(H_{0}+1\right)^{-1 / 2}$ has smoothing properties. We consider the details of this remark in the next lemma and its corollary.

Notation. If $h_{0}$ is a function of type $A$ and if in addition there is an $\alpha>0$ so that $h_{0}(p)>\gamma|p|^{\alpha}$ for some $\gamma>0$ and all $|p|$ sufficiently large, we say that $H_{0}$ is of type $C_{\alpha}$.

Leman 9.3. Let $R_{0}(z)=\left(H_{0}-z\right)^{-1}$ and for $\lambda>0$ let $R_{0}^{1 / 2}(-\lambda)$ be the nonnegative selfadjoint square root of $R_{0}(-\lambda)$. Then if $H_{0}$ is of type $A$, $R_{0}^{1 / 2}(-\lambda)$ is a convolution operator on $L^{2}\left(R^{N}\right)$ with a convolution kernel which is nonnegative and in $L^{1}\left(R^{N}\right)$. Additionally $R_{0}^{1 / 2}(-\lambda)$ extends to a bounded map on $L^{p}\left(\mathbf{R}^{N}\right)$ for $p$ in $[1, \infty]$ with

$$
\left\|R_{0}^{1 / 2}(-\lambda)\right\|_{p, p}<\lambda^{-1 / 2}
$$

Moreover, if $H_{0}$ is of type $C_{\alpha}$ then

$$
\begin{array}{ll}
\left\|R_{0}^{1 / 2}(-\lambda)\right\|_{2, p}<\infty & \text { if } 2<p<2(1-\alpha / N)^{-1}, \alpha<N, \\
\left\|R_{0}^{1 / 2}(-\lambda)\right\|_{2, p}<\infty & \text { if } 2<p<\infty, \alpha>N .
\end{array}
$$

Proof. We use the spectral theorem and the formula

$$
\lambda^{-s}=\Gamma(s)^{-1} \int_{0}^{\infty} t^{s-1} e^{-\lambda t} d t
$$

to write

$$
R_{0}^{1 / 2}(-\lambda)=\pi^{-1 / 2} \int_{0}^{\infty} t^{-1 / 2} e^{-\lambda t} e^{-t H_{0}} d t .
$$

Since $e^{-t H_{0}}(1)=1$, it follows that the convolution kernel of $R_{0}^{1 / 2}(-\lambda)$, which we also denote by $R_{0}^{1 / 2}(-\lambda)$ and which may be defined by 


$$
R_{0}^{1 / 2}(-\lambda)=\pi^{-1 / 2} \int_{0}^{\infty} t^{-1 / 2} e^{-\lambda t} K_{t}^{0} d t,
$$

where $K_{t}^{0}$ is the convolution kernel of $e^{-t H_{0}}$, satisfies $R_{0}^{1 / 2}(-\lambda) * 1=\lambda^{-1 / 2}$ and thus (a) follows as in Theorem 2.3.

Using $\left\|K_{t}^{0}\right\|_{1}=1$ and interpolation we have for $q$ in $[1,2]$ that

$$
\left\|K_{t}^{0}\right\|_{q}<\left(\left\|K_{t}^{0}\right\|_{2}^{2}\right)^{1 / q^{q}}
$$

so that if $h_{0}(p)>\gamma|p|^{\alpha}$ for large $|p|$ then

$$
\left\|K_{t}^{0}\right\|_{q}<c\left(1+t^{-N / \alpha q^{\prime}}\right)
$$

and thus

$$
\left\|R_{0}^{1 / 2}(-\lambda)\right\|_{q}<c \int_{0}^{\infty} t^{-1 / 2} e^{-\lambda t}\left\|K_{t}^{0}\right\|_{q} d t<\infty
$$

if $i$ is in $[1,2]$ and $\frac{1}{2}+N / \alpha q^{\prime}<1$. The inequalities of (b) now follow from Young's inequality.

Corollary 9.4. Let $H_{0}$ be an operator of type $C_{\alpha}$ and let $Z$ be an $H_{0}$-perturbation of type $B$. Let $H=H_{0}+Z$ and set $R(-\lambda)=(H+\lambda)^{-1}$. For $\lambda$ large enough, $t>0$ and $p$ in $[2, \infty]$ satisfying $p^{-1}>\frac{1}{2}(1-\alpha / N)$ there follows

$$
\left\|R(-\lambda)^{1 / 2}\right\|_{2, p}<\infty, \quad\left\|e^{-t H}\right\|_{2, p}<\infty .
$$

Proof. Choose $\lambda$ so that $H+\lambda \geqslant 1$. Then since the domain of $(H+\lambda)^{1 / 2}$ is contained in the domain of $\left(H_{0}+\lambda\right)^{1 / 2}$, the closed graph theorem assures us that

$$
\left\|\left(H_{0}+\lambda\right)^{1 / 2}(H+\lambda)^{-1 / 2}\right\|_{2,2}<\infty
$$

so that $\left\|R(-\lambda)^{1 / 2}\right\|_{2, p}<\left\|R_{0}(-\lambda)^{1 / 2}\right\|_{2, p}\left\|\left(H_{0}+\lambda\right)^{1 / 2}(H+\lambda)^{-1 / 2}\right\|_{2,2}<\infty$. In addition,

$$
\left\|e^{-t H}\right\|_{2, p} \leqslant\left\|R(-\lambda)^{1 / 2}\right\|_{2, p}\left\|(H+\lambda)^{1 / 2} e^{-t H}\right\|_{2,2} .
$$

Notation. Define $\gamma_{\alpha}=\infty$ if $\alpha>N$ and $\gamma_{\alpha}=2(1-\alpha / N)^{-1}$ otherwise.

We now combine the techniques of Theorem 9.1 and Corollary 9.4 to obtain

THEOREM 9.5. Suppose the hypotheses of Theorem 9.1 hold and additionally $H_{0}$ is of type $C_{\alpha}$. Then

$$
\left\|e^{-t H}\right\|_{q, r}<\infty
$$

if $q \in\left[2,2 \lambda_{0}\right]$ and if $r \in\left[q, \gamma_{\alpha} \lambda_{0}\right)$ when $\alpha<N$ and $r \in[q, \infty]$ when $\alpha>N$.

Proof. We use the inequality (9.1) to write 


$$
\left\|e^{-t H_{f}}\right\|_{r}<\left(\left\|e^{-t H_{P}}\right\|_{2, r / p}\right)^{1 / p}\left(\left\|e^{-t H_{0}}\right\|_{q / s, \infty}\right)\|f\|_{q}
$$

for $p$ in $\left[q / 2, \lambda_{0}\right], q$ in $\left[2,2 \lambda_{0}\right]$ and $p / r>\frac{1}{2}(1-\alpha / N)$.

The finiteness of $\left\|e^{-t H_{p}}\right\|_{2, r / p}$ for $r / p$ as above follows from Corollary 9.4.

COROLLARY 9.6. Under the hypotheses of Theorem 9.5 all $L^{2}$ eigenfunctions of $H$ are in $L^{r}$ for $r$ in $\left[2, \gamma_{\alpha} \lambda_{0}\right)$ if $\alpha<N$ and in $L^{r}$ for $r$ in $[2, \infty]$ if $\alpha>N$.

EXAMPLE 9.7. Consider the operator $H=H_{0}+V$ where $H_{0}=-\Delta$ and $V(x)=-\beta|x|^{-2}$ if $|x| \leqslant 1,|V(x)|<c$ for $|x|>1$ and $V$ in $C^{\infty}\left(\mathbf{R}^{N}-\{0\}\right)$. For simplicity we discuss the case $N=3$. Then $V$ is an $H_{0}$-perturbation of type $B$ if and only if $\beta<\frac{1}{4}$ [5]. If $H$ has bound states it is known that the angular momentum zero eigenstates behave like $|x|^{-s}, s=\frac{1}{2}-\left(\frac{1}{4}-\beta\right)^{1 / 2}$ in a neighborhood of the origin [24]. All other eigenstates vanish at the origin and thus all eigenstates of $H$ are in $L^{p}$ for $p<p(\beta)$ where $p(\beta)=6(1-(1-$ $\left.4 \beta)^{1 / 2}\right)^{-1}$. Corollary 9.6 gives the result that all eigenstates of $H$ are in $L^{p}$ for $p<6(4 \beta)^{-1}<p(\beta)$ since $0<\beta<\frac{1}{4}$, which, although not optimal, does give the correct behavior near $\beta=\frac{1}{4}$.

However, in this special case another method of estimation does give the exact result:

For $a$ in $[0,1]$ set $b=1-a$ and $B_{a}=\left(H_{0}+\lambda\right)^{-a} V\left(H_{0}+\lambda\right)^{-b}$. We note that if $\left\|B_{a}\right\|<1$ then

$$
(H+\lambda)^{-1}=\left(H_{0}+\lambda\right)^{-b}\left(1+B_{a}\right)^{-1}\left(H_{0}+\lambda\right)^{-a}
$$

so that

$$
\left\|(H+\lambda)^{-1}\right\|_{2, p}<c\left\|\left(H_{0}+\lambda\right)^{-b}\right\|_{2, p} .
$$

But

$$
\left\|\left(H_{0}+1\right)^{-a} r^{-2}\left(H_{0}+1\right)^{-b}\right\|<\left\|r^{-2 a}\left(H_{0}+1\right)^{-a}\right\|\left\|r^{-2 b}\left(H_{0}+1\right)^{-b}\right\|
$$

and $\left\|r^{-2 a}\left(H_{0}+1\right)^{-a}\right\|$ can be computed exactly for all $a$ [15], giving

$$
\left\|\left(H_{0}+1\right)^{-a} r^{-2}\left(H_{0}+1\right)^{-b}\right\|<\left[4\left((1 / 4)^{2}-(a-1 / 2)^{2}\right)\right]^{-1}
$$

if $\left|a-\frac{1}{2}\right|<\frac{1}{4}$. Thus, for some $\lambda>0,\left\|B_{a}(\lambda)\right\|<1$ if $\gamma<\frac{1}{2}\left(1+\frac{1}{2}(1-\right.$ $4 \beta)^{1 / 2}$ ) and hence, by Sobolev's inequality $\left\|(H+\lambda)^{-1}\right\|_{2, p}<\infty$ if $p<6(1-$ $\left.(1-4 \beta)^{1 / 2}\right)^{-1}$.

Until now we have not imposed any restrictions on the perturbations, $Z$, except those inherent in the definition of type $B$ perturbations. The preceding example shows that without such restrictions the eigenfunctions of $H=H_{0}$ $+Z$ will generally have singularities. In the next theorem we isolate a condition which eliminates these singularities.

THEOREM 9.7. Suppose $H_{0}$ is of type $C_{\alpha}$ and $W$ is the distributional part of an $H_{0}$-perturbation of type B. Fix $\lambda>0$. Suppose that the operator 


$$
Q_{\lambda}=R_{0}^{1 / 2}(-\lambda) W R_{0}^{1 / 2}(-\lambda)
$$

extends to a bounded map on $L^{q}$ for some $q$ satisfying

$$
1<q<2 \text { and } q<(1-\alpha / 2 N)^{-1}
$$

and suppose

$$
\left\|Q_{\lambda}\right\|_{q, Q}<1 \text { and }\left\|Q_{\lambda}\right\|_{2,2}<1 \text {. }
$$

(a) If $V$ is the positive part of an $H_{0}$-perturbation of type $B$ then there exists an $L>0$ such that $\left(H_{0}+V+W+\lambda\right)^{-L}$ is bounded from $L^{2}$ to $L^{\infty}$.

(b) There exists a positive number $\xi=\xi(\alpha, q, N)$ such that if $f$ is in $L^{2}$ then $g=\left(H_{0}+W+\lambda\right)^{-L_{f}}$ satisfies

$$
|g(x)-g(y)|<c|x-y|^{\xi}\|f\|_{2}
$$

Proof. Define $H=H_{0}+W$ and $H_{1}=H_{0}+V+W$. Note that by the Trotter product formula and the approximation theorem the kernel of $e^{-t H}$ is pointwise greater or equal to the kernel of $e^{-t H_{1}}$. The formula $R=(H+\lambda)^{-1}$ $=\int_{0}^{\infty} e^{-t} e^{-t H} d t$ shows that a similar statement is true of the kernels of $(H+\lambda)^{-1}$ and $\left(H_{1}+\lambda\right)^{-1}$. Consequently it suffices to prove the theorem in case $V=0$.

Ths significance of the range of allowable $q$ is that, as was shown in the proof of Lemma 9.3, for $q$ satisfying inequalities $(9.6), R_{0}^{1 / 2}(-\lambda) \in L^{q}$.

Define the function $\gamma:\left[1, q^{\prime}\right] \rightarrow[q, \infty]$ by the formula $\gamma(r)=\left(r^{-1}+q^{-1}\right.$ $-1)^{-1}$. Then by Young's inequality, interpolation and Lemma 9.3, part (a) there is a constant $c$ so that $\left\|R_{0}^{1 / 2}(-\lambda)\right\|_{r, s}<c$ if $1<r<s<\gamma(r)$ and $r<q^{\prime}$ while $\left\|R_{0}^{1 / 2}(-\lambda)\right\|_{r, \infty}<c$ if $r>q^{\prime}$.

Define $\gamma_{0}=1$ and $\gamma_{n}=\gamma\left(\gamma_{n-1}\right)$ for $n=1,2, \ldots, M$ where $M$ is the first positive integer such that $\gamma_{M}>q^{\prime}$. We claim there is a constant $d<\infty$ so that

$$
\left\|R^{M}\right\|_{p, r}<d \quad \begin{aligned}
& \text { if } 1<p<q<r<\infty \text { or } \\
& \text { if } q<p<q^{\prime}, p<r<\infty .
\end{aligned}
$$

To prove (9.7) it suffices to show

$$
\left\|R^{M}\right\|_{1, q}<\infty,\left\|R^{M}\right\|_{q, q}<\infty \text { and }\left\|R^{M}\right\|_{1, \infty}<\infty .
$$

The fact that (9.8) implies (9.7) follows from duality and Riesz-Thorin interpolation.

Let $C=\left(1+Q_{\lambda}\right)^{-1}$ and $R_{0}^{1 / 2}=R_{0}^{1 / 2}(-\lambda)$. Then $R=R_{0}^{1 / 2} C R_{0}^{1 / 2}$ so that

$$
\|R\|_{q, q}<\left\|R_{0}^{1 / 2}\right\|_{q, q}^{2}\|C\|_{q, q}, \quad\|R\|_{1, q}<\left\|R_{0}^{1 / 2}\right\|_{1, q}\|C\|_{q, q}\left\|R_{0}^{1 / 2}\right\|_{q, q}
$$

These two inequalities prove the first two inequalities in (9.8).

To show that $\left\|R^{M}\right\|_{1, \infty}<\infty$ we use 


$$
\begin{aligned}
\left\|R^{M}\right\|_{1, \infty}< & \left\|R_{0}^{1 / 2}\right\|_{1, \gamma_{1}} \cdot\|C\|_{\gamma_{1}, \gamma_{1}} \cdot\left\|R_{0}^{1 / 2}\right\|_{\gamma_{1}, \gamma_{2}} \\
& \cdot\left\|R_{0}^{1 / 2}\right\|_{\gamma_{2}, \gamma_{2}} \cdot\|C\|_{\gamma_{2}, \gamma_{2}}\left\|R_{0}^{1 / 2}\right\|_{\gamma_{2}, \gamma_{3}} \cdot \cdots \\
& \cdot\left\|R_{0}^{1 / 2}\right\|_{\gamma_{M-1}, \gamma_{M-1}}\|C\|_{\gamma_{M-1}, \gamma_{M-1}}\left\|R_{0}^{1 / 2}\right\|_{\gamma_{M-1}, \gamma_{M}} \\
& \cdot\left\|R_{0}^{1 / 2}\right\|_{\gamma_{M}, q^{\prime}}\|C\|_{q^{\prime}, q^{\cdot}}\left\|R_{0}^{1 / 2}\right\|_{q^{\prime}, \infty}<\infty
\end{aligned}
$$

where the fact that $\|C\|_{p, p}<c^{\prime}<\infty$ for $p$ in $\left[q, q^{\prime}\right]$ follows from duality and interpolation. (9.7) proves part (a) with $L=M$. $L^{q}$

The proof of part (b) will consist of verifying the following estimate for $h$ in

$$
\left|\left(R_{0}^{1 / 2} h\right)(x)-\left(R_{0}^{1 / 2} h\right)(y)\right|<c|x-y|^{\xi}\|h\|_{q^{\prime}}
$$

since if $f \in L^{2}$ we can write $R^{L} f=R_{0}^{1 / 2} h$ where $h=C R_{0}^{1 / 2} R^{L-1} f$. It is clear from a glance at (9.9) that $\left\|R^{L-1}\right\|_{2, q^{\prime}}<\infty$ so that $\|h\|_{q^{\prime}}<C\|f\|_{2}$. Here we have assumed $L>1$ but this involves no loss of generality since $\left\|R^{L+1}\right\|_{1, \infty}$ $<\infty$ providing $\left\|R^{L}\right\|_{1, \infty}<\infty$. The proof of $(9.10)$ will involve

LeMMa 9.8. Suppose $H_{0}$ is of type $C_{\alpha}$. Then for $t>0$ the convolution kernel, $K_{l}^{0}$, of $e^{-t H_{0}}$ is in $C^{\infty}\left(\mathbf{R}^{N}\right)$ and there are nonnegative constants $A, \beta, l$, with $\beta$ and $l$ functions of $\alpha$ alone, such that

$$
\left\|\nabla K_{t}^{0}\right\|_{1}<A t^{-\beta}\left(1+t^{t}\right)
$$

Proof. We have

$$
K_{t}^{0}(x)=(2 \pi)^{-N} \int e^{i x \cdot p} e^{-t h_{0}(p)} d^{N} p
$$

which is clearly in $C^{\infty}$. By Lemma $2.2, h_{0}$ is given by the Levy-Khintchine formula with some finite measure $\mu$. Let

$$
h_{1}(p)=\frac{1}{2} \int_{1>|\lambda|>0}\left(1+\lambda^{2}\right)(\lambda)^{-2}(1-\cos (\lambda \cdot p)) d \mu(\lambda)+\frac{1}{2}(p, C p)
$$

and

$$
h_{2}(p)=h_{0}(p)-h_{1}(p) .
$$

It is clear that if $H_{i}=\mathscr{F} M_{h_{i}} \mathscr{F}^{-1}$, then each $H_{i}$ is also of type $C_{\alpha}$ for $i=1,2$. If $e^{-t H_{i}}$ is given by convolution with $K_{t}^{i}$ then we have

$$
K_{t}^{0}=K_{t}^{1} * K_{t}^{2}
$$

so that $\nabla K_{t}^{0}=\nabla K_{t}^{1} * K_{t}^{2}$ satisfies

$$
\left\|\nabla K_{t}^{0}\right\|_{1}<\left\|\nabla K_{t}^{1}\right\|_{1} \text {. }
$$

Thus it suffices to consider $H_{0}$ of type $C_{\alpha}$ for which the measure in the Lévy-Khintchine formula is supported on a bounded subset of $\mathbf{R}^{N}$. For such $H_{0}$, the gradient of the $K_{t}^{0}$ of (9.11) can be estimated explicitly. We use the 
fact that

$$
\left|\nabla h_{0}\right|^{2}<c h_{0}
$$

as proved in Lemma 2.7 to conclude that for $|n|>2$

$$
\left|D^{(n)} e^{-t h_{0}}\right|<c(n) t\left(1+t^{|n|}\right) e^{-t h_{0} / 2} \text {. }
$$

Thus integrating by parts we find for $|n| \geqslant 3$

$$
\begin{aligned}
\left|\left(\nabla K_{t}^{0}\right)(x)\right| & <b_{n}|x|^{-n} t\left(1+t^{n}\right) \int e^{-t h_{0} / 2}(1+|p|) d^{N} p \\
& <a_{n}|x|^{-n} t^{1-(N+1) / \alpha}\left(1+t^{n}\right) .
\end{aligned}
$$

Consequently,

$$
\left\|\nabla K_{t}^{0}\right\|_{1}<c\left\|\nabla K_{t}^{0}\right\|_{2}+c^{\prime}\left(t^{(1-(N+1) / \alpha)}\right)\left(1+t^{N+1}\right)
$$

where the first term comes from estimating $\int_{|x|<1 \mid}\left|\nabla K_{l}^{0}\right|(x) d^{N} x$. The lemma follows by explicitly estimating $\left\|\nabla K_{t}^{0}\right\|_{2}$ using the Plancherel theorem.

REMARK. In special cases our estimate for $\beta(\alpha)$ is poor. For example, if $h_{0}(p)=|p|^{\alpha}$ then, by scaling, $\left\|\nabla K_{t}^{0}\right\|_{1}=c t^{-1 / \alpha}$. A better estimate for $\beta(\alpha)$ leads to a better estimate on the modulus of Hölder continuity in Theorem 9.7.

We now complete the proof of Theorem 9.7 by proving (9.10).

Suppose $h \in L^{q^{\prime}}$. Then

$$
\left|\left(K_{t}^{0} * h\right)(x)-\left(K_{t}^{0} * h\right)(y)\right|<\left\|K_{t}^{y-x}-K_{t}^{0}\right\|_{q}\|h\|_{q^{\prime}}
$$

where $K_{l}^{x}(z)=K_{t}^{0}(z+x)$. Now

$$
K_{t}^{x}(z)-K_{t}^{0}(z)=\int_{0}^{1} x \cdot \nabla K_{t}^{0}(z+s x) d s
$$

so that

$$
\left\|K_{t}^{x}-K_{t}^{0}\right\|_{q}<|x|\left\|\nabla K_{t}^{0}\right\|_{q}
$$

Since $q \in[1,2]$ we have

$$
\left\|\nabla K_{t}^{0}\right\|_{q}<\left\|\nabla K_{t}^{0}\right\|_{1}^{s}\left\|\nabla K_{t}^{0}\right\|_{2}^{1-s}
$$

where $1 / q=s / 1+(1-s) / 2$ which gives, for some $\beta_{1}>\frac{1}{2}$,

$$
\left\|K_{t}^{x}-K_{t}^{0}\right\|_{q} \leqslant c_{1}|x| t^{-\beta_{1}}\left(1+t^{N_{1}}\right) \text {. }
$$

We also have

$$
\left\|K_{t}^{x}-K_{t}^{0}\right\|_{q}<2\left\|K_{t}^{0}\right\|_{q} \leqslant c_{2} t^{-\beta_{2}}\left(1+t^{\beta_{2}}\right)
$$

from (9.3) where $0<\beta_{2}<\frac{1}{2}$.

If we express the region of integration in the inequality 


$$
\left|\left(R_{0}^{1 / 2} h\right)(x)-\left(R_{0}^{1 / 2} h\right)(y)\right|<\left(c \int_{0}^{\infty} t^{-1 / 2} e^{-\lambda t}\left\|K_{l}^{y-x}-K_{t}^{0}\right\|_{q} d t\right)\|h\|_{q^{\prime}}
$$

as the union of $[0, s]$ and $(s, \infty)$, then using $(9.15)$ in $[0, s]$ and $(9.14)$ in $(s, \infty)$ gives (9.10) with $\xi=\left(\frac{1}{2}-\beta_{2}\right) /\left(\beta_{1}-\beta_{2}\right)$ after optimizing the power $p$ in $s=|x-y|^{p}$.

This completes the proof of Theorem 9.7.

REMARK. If $H_{0}=-\Delta$ in $\mathbf{R}^{N}$ then by scaling, $\left\|\nabla K_{t}^{0}\right\|_{q}=c_{1} t^{-(1 / 2)(1+N / q)}$ and $\left\|K_{t}^{0}\right\|_{q}=c_{2} t^{-N / 2 q^{\prime}}$. The procedure used in the proof of Theorem 9.7 gives $\xi=1-N / q^{\prime}$.

EXAMPLE 9.9. Suppose $H_{0}=-\Delta$ in $\mathbf{R}^{N}, N>1$, and suppose $W$ is a real distribution with $W=\sum_{j=1}^{N} \partial_{j} V_{j}+V$ where $V_{j} \in L^{p}, p>N$, and $V \in L^{q}+$ $L^{\infty}, q \in(N / 2, \infty)$. Then $W$ satisfies the hypotheses of Theorem 9.7.

For the proof of this statement first note that without loss of generality we may assume $V \in L^{q}$ and $V>0$. Then

$$
|W(f, g)|<\sum_{j}\left\|V_{j}\right\|_{p}\left(\left\|\partial_{j} f\right\|_{r}\|g\|_{s}+\left\|\partial_{j} g\right\|_{u}\|f\|_{0}\right)+\left\|V^{1 / 2} f\right\|_{r}\left\|V^{1 / 2}\right\|_{\|_{r}}
$$

where $p^{-1}+r^{-1}+s^{-1}=u^{-1}+v^{-1}+p^{-1}=r^{-1}+r^{-1}=1$. Since $\left\|\partial_{j}(-\Delta+\lambda)^{-1 / 2}\right\|_{q . q}<c(q)<\infty$, independent of $\lambda$ for $q$ in $(1, \infty)$ [34], we have

$$
\begin{aligned}
\left|W\left(R_{0}^{1 / 2}(-\lambda) f, R_{0}^{1 / 2}(-\lambda) g\right)\right| \\
<c\left(\|f\|_{r}\left\|R_{0}^{1 / 2}(-\lambda) g\right\|_{s}+\|g\|_{2}\left\|R_{0}^{1 / 2}(-\lambda) f\right\|_{v}\right) \\
+\left\|V^{1 / 2} R_{0}^{1 / 2}(-\lambda) f\right\|_{r}\left\|V^{1 / 2} R_{0}^{1 / 2}(-\lambda) g\right\|_{r^{\prime}}
\end{aligned}
$$

Suppose $r \in\left[(2 q)^{\prime}, 2 q\right]$. Then by Young's inequality and Hölder's inequality $\left\|V^{1 / 2} R_{0}^{1 / 2}(-\lambda)\right\|_{r, r}$ and $\left\|V^{1 / 2} R_{0}^{1 / 2}(-\lambda)\right\|_{r, r}$ approach zero as $\lambda \rightarrow$ $\infty$. Also, $\left\|R_{0}^{1 / 2}(-\lambda)\right\|_{r^{\prime},}$ and $\left\|R_{0}^{1 / 2}(-\lambda)\right\|_{u^{\prime}, 0}$ approach zero as $\lambda \rightarrow \infty$ by Young's inequality. Taking $u=r^{\prime}$ we thus have

$$
\lim _{\lambda \rightarrow \infty} \sup _{\|\|_{r}-\|\|_{r^{\prime}-1}}\left|\left(f, R_{0}^{1 / 2}(-\lambda) W R_{0}^{1 / 2}(-\lambda) g\right)\right|=0
$$

so that for $\lambda$ sufficiently large

$$
\left\|R_{0}^{1 / 2}(-\lambda) W R_{0}^{1 / 2}(-\lambda)\right\|_{t, t}<1
$$

if $t \in\left[(2 q)^{\prime}, 2 q\right]$.

EXAMPLE 9.10. If $H_{0}=-d^{2} / d x^{2}$ in $\mathbf{R}^{1}$ then any distributional part, $W$, of an $H_{0}$-perturbation of type $B$ satisfies the hypotheses of Theorem 9.7.

REMARK. Smoothness properties of the eigenfunctions of $N$-body Schrödinger Hamiltonians have been investigated by Kato [23] and Simon [33]. These authors are mainly interested in the case where the perturbations are sums of two-body potentials which are relatively bounded with respect to 
$H_{0}=-\Delta$ in $\mathbf{R}^{3}$. With these stronger hypotheses, the eigenfunctions are smoother than the result given by Theorem 9.7.

EXAMPLE 9.11. Let $H_{0}=-\Delta$ in $\mathbf{R}^{N}, N>1$. Let $\delta_{F}$ be a delta function concentrated on a compact $C^{1}$ hypersurface, $S=F^{-1}(\{0\})$ as in Example 4.6. If $W$ is the associated form then $W$ satisfies the hypotheses of Theorem 9.7.

To prove this we will use the inequality

$$
\left\|\sup _{x_{1}}\left|\left(R_{0}^{1 / 2}(-\lambda) f\right)\left(x_{1}, \cdot\right)\right|\right\|_{L^{q}\left(R^{N-1}, d^{N-1} x\right)}<c_{q} \lambda^{-1 / 2 q^{\prime}}\|f\|_{q}
$$

for $q$ in $(1, \infty), f \in \delta\left(\mathbf{R}^{N}\right)$, which we will shortly prove. After decomposing $S$ into finitely many pieces which can be straightened out, $(9.16)$ is easily seen to give

$$
|W(f, g)|<\beta_{q} \lambda^{-1 / 2}\left\|(-\Delta+\lambda)^{1 / 2}\right\|_{q}\left\|(-\Delta+\lambda)^{1 / 2} g\right\|_{q^{\circ}}
$$

Thus

$$
\left\|R_{0}^{1 / 2}(-\lambda) W R_{0}^{1 / 2}(-\lambda)\right\|_{q, q}<\beta_{q} \lambda^{-1 / 2}
$$

for $q$ in $(1, \infty)$.

All that remains is to verify $(9.16)$ which we now do.

First observe that from $\left\|R_{0}^{1 / 2}(-\lambda)\right\|_{q}<c(q) \lambda^{-1 / 2 q}$ and Hölder's inequality there follows

$$
\left\|R_{0}^{1 / 2}(-\lambda) f\right\|_{\infty}<c\left(q^{\prime}\right) \lambda^{-1 / 2 q^{\prime}}\|f\|_{q^{\prime}}, \quad N=1 .
$$

If $f \in \delta\left(\mathbf{R}^{N}\right)$ then (9.17) implies

$$
\begin{aligned}
\sup _{x_{1}}\left|\left(\left(p_{1}^{2}+\lambda\right)^{-1 / 2} f\right)\left(x_{1}, \ldots, x_{N}\right)\right| \\
<c\left(q^{\prime}\right) \lambda^{-1 / 2 q^{\prime}}\left(\int\left|f\left(x_{1}, \ldots, x_{N}\right)\right|^{q} d x_{1}\right)^{1 / q}
\end{aligned}
$$

which in turn implies

$$
\left\|\sup _{x_{1}} \mid\left(\left(p_{1}^{2}+\lambda\right)^{-1 / 2} f\right)\left(x_{1}, \cdot\right)\right\|\left\|_{L^{9}\left(\mathbb{R}^{N-1}, d x^{N-1}\right)}<c\left(q^{\prime}\right) \lambda^{-1 / 2 q^{\prime}}\right\| f \|_{q^{\prime}}
$$

Let $R_{0}^{1 / 2}(-\lambda) g=\left(p_{1}^{2}+\lambda\right)^{-1 / 2}$. Then from (9.19)

$$
\begin{aligned}
\| \sup _{x_{1}} \mid\left(R_{0}^{1 / 2}(-\lambda) g\right)\left(x_{1}, \cdot\right) & \|_{L^{q}\left(\mathrm{R}^{N-1}\right)} \\
& <c\left(q^{\prime}\right) \lambda^{-1 / 2 q^{\prime}}\left\|\left(p_{1}^{2}+\lambda\right)^{1 / 2}\left(p^{2}+\lambda\right)^{-1 / 2} g\right\|_{q}
\end{aligned}
$$

Now it follows from the equivalence of the norms $\left(\sum_{i=1}^{N}\left\|\partial_{i} g\right\|_{q}+\|g\|_{q}\right)$ and $\left\|\left(p^{2}+1\right)^{1 / 2} g\right\|_{q}$ as shown in [34] for $q$ in $(1, \infty)$ that $\|\left(p_{1}^{2}+\lambda\right)^{1 / 2}\left(p^{2}+\right.$ 
$\lambda)^{-1 / 2} \|_{q, q}=c(q, \lambda)<\infty$. The fact that $c(q, \lambda)$ is independent of $\lambda$ follows by a scaling argument.

$X$. Generalized eigenfunctions. While many selfadjoint operators have no eigenfunctions in the Hilbert space sense they may possess generalized eigenfunctions. For example

$$
-d^{2}\left(e^{i k x}\right) / d x^{2}=k^{2} e^{i k x}
$$

and if $V$ is multiplication by the coordinate function on $L^{2}\left(R^{1}\right)$ then $V \delta=0$ in the sense of distributions but $V$ has no eigenfunctions in the $L^{2}\left(R^{1}\right)$ sense. Here $\delta$ is the delta function. Generalized eigenfunctions, such as $\delta$ and $e^{i k x}$, are useful in giving mathematical meaning to common heuristic expressions (see [8] for example) such as

$$
H|g\rangle=\int H|\xi\rangle\langle\xi \mid g\rangle d \xi
$$

where the $|\xi\rangle$ form a complete set of eigenvectors for the observable $H$.

We begin this section with a review of results concerning generalized eigenfunction expansions of selfadjoint operators. There are various approaches and an extensive literature on this subject, see for example [1], [3], [9], [13], [17], [18], [22], [25], [27], and [35]. We simultaneously consider the question of when the generalized eigenfunctions are functions rather than more general distributions. We end this section by discussing the singularity structure of the generalized eigenfunctions in the sense that we describe functions $\rho$ such that the generalized eigenfunctions belong to $L^{2}(\rho d x)$.

In this section $T$ will denote a selfadjoint operator on a separable Hilbert space $L^{2}(X, d \mu)$ where $\mu$ is a $\sigma$-finite and positive measure. By the spectral theorem there is a $\sigma$-finite positive measure space $(Z, d v)$, a real measurable function $\alpha$ on $Z$ and a unitary operator $U: L^{2}(X, d \mu) \rightarrow L^{2}(Z, d \nu)$ such that $U H U^{-1}$ is multiplication by $\alpha$.

If $v$ and $w$ are measurable functions on some measure space we will write $\langle v, w\rangle$ for the integral of $\bar{v} w$ in case $\bar{v} w$ is integrable.

Definition. A selfadjoint operator $S$ on $L^{2}(X, d \mu)$ is called a Carleman operator if $S$ is a bounded operator with kernel satisfying

$$
\int|S(x, y)|^{2} d \mu(y)<\infty \text { for almost every } x .
$$

A weight function for a Carleman operator $S$ is a nonnegative measurable function $\rho$ on $X$ such that

$$
\iint|S(x, y)|^{2} \rho(y) d \mu(y) d \mu(x)<\infty .
$$

Observe that positive weight functions always exist for Carleman operators $S$. In fact if $\gamma>0$ is in $L^{1}(X, d \mu)$, setting 


$$
\rho(y)= \begin{cases}\left(\int|S(x, y)|^{2} d \mu(x)\right)^{-1} \gamma(y) & \text { if } 0<\int|S(x, y)|^{2} d \mu(x)<\infty, \\ 1 & \text { otherwise, }\end{cases}
$$

gives a positive weight function for $S$.

Definimion. A Carleman function for the selfadjoint operator $T$ is a Borel function $\beta$ which does not vanish on the spectrum of $T$ and for which $\beta(T)$ is a Carleman operator.

Notation. $\Gamma_{+}=\{\rho: \rho$ is a nonnegative measurable function on $X\}, \Gamma_{T}=\{\rho$ $\in \Gamma_{+}: M_{\rho^{1 / 2}} \beta(T)$ is a Hilbert Schmidt operator for some bounded Borel function $\beta$ which is nonvanishing on the spectrum of $T$ \}.

We remark that $\Gamma_{T}$ is a convex cone so that

$$
V_{T} \equiv \bigcup_{\rho \in \Gamma_{T}} L^{2}\left(X, \rho^{-1} d \mu\right)
$$

is a linear space. (Note that we allow the set $E=\{x: \rho(x)=0\}$ to have positive measure. In this case $L^{2}\left(X, \rho^{-1} d \mu\right) \equiv$ set of all $f$ with $\chi_{E} f=0$, $\int_{X \backslash E}|f|^{2} \rho^{-1} d \mu<\infty$.)

Minor modifications of techniques developed in [1] and [9] yield the following generalization of results found there:

THEOREM 10.1. Suppose $\Gamma_{T}$ contains an a.e. positive function. Then there is a set of generalized eigenfunctions $\left\{\psi_{z}: z \in Z\right\}$ such that

(a) For all $\rho \in \Gamma_{T}$, almost every generalized eigenfunction is in $L^{2}(X, \rho d \mu)$, i.e. $\psi_{z} \in L^{2}(X, \rho d \mu)$ for $\nu$-almost every $z$.

$$
(U h)(z)=\left\langle\psi_{z}, h\right\rangle \text { for all } h \in V_{T} \cap L^{2}(X, d \mu) .
$$

(c) If $\gamma$ is a bounded Borel function then

$$
(f, \gamma(T) g)=\int\left\langle f, \psi_{z}\right\rangle \gamma(\alpha(z))\left\langle\psi_{z}, g\right\rangle d \nu(z)
$$

for all $f, g \in V_{T} \cap L^{2}(X, d \mu)$.

Remarks. (a) $V_{T} \cap L^{2}(X, d \mu)$ is dense in $L^{2}(X, d \mu)$.

(b) The statement that $\Gamma_{T}$ contains an a.e. positive function is equivalent to the statement that there exists a Carleman function for the operator $T$.

(c) Since $U$ may not be an integral operator, as in the case of the Fourier transform, (10.3) cannot in general be extended to all of $L^{2}(X, d \mu)$. However in this direction we do have

COROLLARY 10.2. Let $\left\{E_{n}\right\}$ be an increasing sequence of $\mu$-measurable subsets of $X$ with $\cup_{n} E_{n}=X$. Suppose each characteristic function, $\chi_{n}$, of $E_{n}$ belongs to $\Gamma_{T}$. Then for all $h \in L^{2}(X, d \mu)$ 


$$
U h=L^{2}-\lim _{n \rightarrow \infty}\left\langle\psi, x_{n} h\right\rangle .
$$

Proof. By (10.3), $U\left(\chi_{n} h\right)=\left\langle\psi, \chi_{n} h\right\rangle$ and $\chi_{n} h \rightarrow^{L^{2}} h$. Q.E.D.

Note that if $\rho>0$ is in $\Gamma_{T}, E_{n}=\{x: 1 / n<\rho(x)\}$ satisfies the hypotheses of the corollary.

We now turn our attention to the case of $X=L^{2}\left(\mathrm{R}^{N}, d x\right)$ and $T=H_{0}+Z$ where now and for the rest of this section $H_{0}$ denotes an operator of type $A$ and $Z$ denotes an $H_{0}$-perturbation of type $B$ with distributional part $W$ and positive part $V$. Our first goal is to show that $H_{0}+Z$ has a set of generalized eigenfunctions. By Theorem 10.1 this goal will be attained upon finding a Borel function $\beta$, not vanishing on the spectrum of $H_{0}+Z$, such that $\beta\left(H_{0}+Z\right)$ is a Carleman operator. Secondly we shall describe functions $\rho$ such that the generalized eigenfunctions are in $L^{2}\left(\mathbf{R}^{N}, \rho d x\right)$. Here the results may be compared to the case of $-\Delta$ where the generalized eigenfunctions, $e^{-i k \cdot x}$, are in $L^{2}\left(\mathbf{R}^{N}, \rho d x\right)$ for all $\rho$ in $L^{1}\left(\mathbf{R}^{N}, d x\right)$. See also [9]. With reference to such a comparison a most satisfactory result (and recalling the examples following Theorem 9.7, an interesting result) is contained in

THEOREM 10.3. Let $H_{0}, \lambda$ and $Z$ satisfy the hypotheses of Theorem 9.7. Then there is a positive integer $L$ such that $\left(H_{0}+Z+\lambda\right)^{-L}$ is a Carleman operator.

Moreover, $L^{1} \subseteq \Gamma_{H_{0}+Z}$ and thus for every $\rho$ in $L^{1}\left(\mathbf{R}^{N}, d x\right)$ almost every generalized eigenfunction of $H_{0}+Z$ is in $L^{2}\left(\mathrm{R}^{N}\right.$, $\left.\rho d x\right)$.

Proof. Let $S=\left(H_{0}+Z+\lambda\right)^{-L}$ where $L$ is as in Theorem 9.7. From inequality (9.7) of the proof of Theorem 9.7 integration against the kernel of $S$ gives a bounded map of $L^{1}$ into $L^{\infty}$ and by the Dunford-Pettis theorem [37] the kernel of $S$ is bounded, say by the constant $c$. Let $\rho$ be a nonnegative function in $L^{1}\left(R^{N}, d x\right)$. Then

$$
\begin{aligned}
\iint|S(x, y)|^{2} \rho(y) d y d x & <c \iint S(x, y) \rho(y) d y d x \\
& =c\|S(\rho)\|_{1}<c\|S\|_{1,1}\|\rho\|_{1} .
\end{aligned}
$$

Again from (9.7), $\|S\|_{1,1}<\infty$. This completes the proof of Theorem 10.3.

Remarks. (a) This theorem and Example 9.9 generalize a result of Faris [9].

(b) For $H_{0}$ and $Z$ as in the previous theorem, we may conclude that $e^{-t\left(H_{0}+Z\right)}$ is a Carleman operator for all $t>0$.

(c) If $H_{0}$ and $Z$ satisfy the hypothesis of Theorem 10.3 then the characteristic function of any measurable subset of $\mathbf{R}^{N}$ with finite Lebesgue measure belongs to $W_{\beta\left(H_{0}+z\right)}$ where $\beta(x)=(x+\lambda)^{-L}$. Consequently, Corollary 10.2 is applicable in this case.

(d) From Theorem 2.3 it may be seen that $e^{-t_{0}}$ is the Fourier transform of an $L^{1}$ function which implies that $e^{-t h_{0}}$ vanishes at $\infty$. The hypothesis of 
Theorem 10.3 which requires $H_{0}$ to be of type $C_{\alpha}$ restricts the rate of vanishing.

Since the convolution kernel, $K_{t}^{0}$, of $e^{-t h_{0}}$ is the Fourier transform of the $L^{1}$ function $e^{-t h_{0}}$, it likewise vanishes at $\infty$. We next consider a class of $H_{0}$ 's for which this decrease property is made more quantitative.

Definition. A nonnegative function, $f$, is said to be decreasing with respect to the norm $\|\cdot\|$, if $f(x)<f(y)$ whenever $\|x\|>\|y\|$.

Let $D \equiv\left\{f>0: f(x)<g(x)\right.$ for some $g$ in $L^{2}$ which is decreasing with respect to the norm $\|\cdot\|\}$.

We remark that $D$ is independent of the norm, $\|\cdot\|$, which we use to define it.

Defintion. $H_{0}$ is of type $D$ if it is of type $A$ and in addition $K_{t}^{0}$ is in $D$ for all $t>0$.

We will soon obtain eigenfunction expansions for a large class of perturbed $H_{0}$ 's of type $D$. For now we note that this class is quite large.

Proposition 10.4. Suppose $h_{0}$ is of type A. Each of the following is a sufficient condition for $H_{0}=\mathscr{F} M_{h_{0}} \mathscr{F}^{-1}$ to be of type $D$ :

(i) $h_{0} \in C^{\infty}\left(\mathbf{R}^{n}\right)$.

(ii) $H_{0}=H_{1}+H_{2} ; H_{1}, H_{2}$ are of type $D$.

(iii) $H_{0}=g \circ h_{1}$, where $h_{1}$ is of type $A, F_{e^{-t h_{1}}}$ is for each $t>0$ decreasing with respect to a fixed norm, and $g$ is a function of the type discussed after Example 2.6.

The proof of this proposition is given at the end of this section.

REMARKS. (a) It is an interesting fact (which we include in our proof of Proposition 10.4) that if $h_{0}$ is of type $A$ and is $C^{\infty}$ in a neighborhood of zero, then $h_{0} \in C^{\infty}\left(\mathbf{R}^{n}\right)$.

(b) Since $e^{t \Delta}$ has a decreasing convolution kernel, condition (iii) above gives many examples of $H_{0}$ 's of type $D$. For example $H_{0}=\mathscr{F} M_{|p|^{\alpha}} \mathscr{F}^{-1}$ for $0<\alpha<2$.

THEOREM 10.5. Suppose $H_{0}$ is of type $D$ and that $2 Z$ is an $H_{0}$-perturbation of type $B$.

Then $e^{-t\left(H_{0}+Z\right)}$ is a Carleman operator for each $t>0$.

Remark. Deift and Simon [7] have proved Theorem 10.5 with $H_{0}=-\Delta$ assuming only that $Z$ is an $H_{0}$ perturbation of type $B$. Their method is very different from ours and uses techniques which are special to $H_{0}=-\Delta$. However it is not all clear that Theorem 10.5 cannot be improved in general.

Proof. $p Z$ is an $H_{0}$-perturbation of type $B$ for some $p>2$. Let $\beta=(p-$ 1) $(p-2)^{-1}$. Let $\rho$ be a positive function in $L^{1} \cap L^{\beta}\left(\mathbf{R}^{N}\right)$ which is decreasing with respect to some norm. Let $K_{r}$ be the kernel of $e^{-t H_{r}}$ where $H_{r}=H_{0}+$ 
$r Z, 0<r<p$. From Theorem 8.2 we have

$$
\begin{aligned}
\iint\left|K_{1}(x, y)\right|^{2} \rho(y) d y d x & \\
< & \iint\left(K_{0}(x, y) \rho^{1 / 2}(y)\right)^{2 / p^{\prime}}\left(K_{p}(x, y) \rho^{1 / 2}(y)\right)^{2 / p} d y d x \\
< & \int d x\left\{\left[\int\left(K_{0}(x, y) \rho^{1 / 2}(y)\right)^{[(2 p-2) / p] \cdot p / p-2} d y\right]^{(p-2) / p}\right. \\
\cdot & {\left.\left[\int K_{p}(x, y) \rho^{1 / 2}(y) d y\right]^{2 / p}\right\} } \\
< & {\left[\int d x\left(\int d y K_{0}(x, y)^{2 \beta} \rho(y)^{\beta}\right)^{1 / \beta}\right]^{1 / p^{\prime}} \| e^{-t H_{\rho} \rho^{1 / 2} \|_{2}^{2 / p}} . }
\end{aligned}
$$

The first factor is controlled using our assumptions on $K_{t}^{0}$ and $p$ : we have $K_{0}(x, y)<g(x-y)$ with $g \in D$ so that

$$
\begin{aligned}
\int K_{0}(x, y)^{2 \beta} \rho(y)^{\beta} d y< & \int_{\|y\|<1 / 2\|x\|} g(x-y)^{2 \beta} \rho^{\beta}(y) d y \\
& +\int_{\|y\|>1 / 2\|x\|} K_{0}(x, y)^{2 \beta} \rho^{\beta}(y) d y \\
& \leqslant g\left(\frac{1}{2} x\right)^{2 \beta}\|\rho\|_{\beta}^{\beta}+\rho^{\beta}(\gamma x)\left\|K_{t}^{0}\right\|_{2 \beta}^{2 \beta}
\end{aligned}
$$

so that

$$
\begin{aligned}
\int d x\left(\int d y\right. & \left.K_{0}(x, y)^{2 \beta} \rho(y) d y\right)^{1 / \beta} \\
& <c_{1}\left\{\|g\|_{2}^{2}\|\rho\|_{\beta}+\left\|K_{t}^{0}\right\|_{2 \beta}^{2}\|\rho\|_{1}\right\} \leqslant c_{2}\left(\|\rho\|_{\beta}+\|\rho\|_{1}\right) .
\end{aligned}
$$

The second factor is controlled with the estimate

$$
\left\|e^{-t H_{\rho} \rho} \rho^{1 / 2}\right\|_{2}<\|\rho\|_{1}^{1 / 2}\left\|e^{-t H_{\rho}}\right\|_{2,2}
$$

so that finally we have

$$
\int\left|K_{1}(x, y)\right|^{2} \rho(y) d y d x<c(t)\|\rho\|_{1}^{1 / p}\left(\|\rho\|_{\beta}+\|\rho\|_{1}\right)^{1 / \rho^{\prime}}
$$

which completes the proof.

We now consider the set $\Gamma_{H_{0}+Z}$ and thus to some extent the singularity structure of the generalized eigenfunctions.

Notation. $L_{c}^{p}\left(\mathbf{R}^{N}\right)=L^{p}\left(\mathbf{R}^{N}\right) \cap\{f: \operatorname{supp} f$ is compact $\}$.

Proposition 10.6. Suppose $H_{0}$ is of type $D$ and $\lambda_{0} Z$ is an $H_{0}$-perturbation of 
type $B$ for some $\lambda_{0}>2$. Then

$$
\left\{\rho \geqslant 0: \rho \in L_{c}^{\lambda_{0}^{\prime}}\left(\mathbf{R}^{N}\right)\right\} \subseteq \Gamma_{H_{0}+z}
$$

and thus if $\rho>0, \rho \in L_{c}^{\lambda_{0}^{\prime}}$, then almost every generalized eigenfunction of $H_{0}+Z$ is in $L^{2}\left(\mathrm{R}^{N}, \rho d x\right)$.

REMARKs. (a) Corollary 9.2 shows that under the hypotheses of Proposition 10.6 every eigenfunction is in $L^{2 \lambda_{0}}\left(\mathbf{R}^{N}\right)$ and thus in $L^{2}(\rho d x)$ for every nonnegative $\rho \in L^{\lambda_{0}^{\prime}}\left(\mathbf{R}^{N}\right)$. Thus Proposition 10.6 should be considered a local version of Corollary 9.2 for generalized eigenfunctions.

(b) The proof of Theorem 10.5 shows that $\left\{\rho>0: \rho \in L^{1} \cap L^{\beta}, \beta=\lambda_{0}\right.$ $-1)\left(\lambda_{0}-2\right)^{-1}, \rho(x-a)=h(x)$ decreasing with respect to some norm $\} \subseteq$ $\Gamma_{H_{0}+z}$. However, since $\beta>\lambda_{0}^{\prime}$, Proposition 10.6 is a better result locally.

Proof. We use the notation of Theorem 10.5. With $p=\lambda_{0}$ we have

$$
\begin{aligned}
& \iint\left|K_{1}(x, y)\right|^{2} \rho(y) d x d y \\
&< \int d x \int d y K_{0}(x, y)^{2 / p^{\prime}} \rho(y)^{1 / \beta}\left(K_{p}(x, y) \rho(y)^{p^{\prime} / 2}\right)^{2 / p} \\
&< {\left[\int d x\left(\int d y K_{0}(x, y)^{2 \beta} \rho(y)^{p^{\prime}}\right)^{1 / \beta}\right]^{1 / p^{\prime}} } \\
& \cdot\left[\int d x\left(\int K_{p}(x, y) \rho(y)^{p^{\prime} / 2} d y\right)^{2}\right]^{1 / p}
\end{aligned}
$$

The second factor is dominated by $\left\|e^{-t H_{p}}\right\|_{2,2}^{2 / p}\|\rho\|_{p^{\prime}}^{p^{\prime} / p}$ while the first is controlled using the fact that $H_{0}$ is of type $D$ and $\rho(x)$ vanishes for $\|x\|>c$. Thus note that if $K_{0}(x, y)<g(x-y)$ where $g \in D$ we have

$$
\left(\int d y K_{0}(x, y)^{2 \beta} \rho(y)^{p^{\prime}}\right)^{1 / \beta}<\left\|K_{0}\right\|_{\infty}^{2}\|\rho\|_{p^{\prime}}^{p^{\prime} / \beta} \text { for } x \text { arbitrary, }
$$

and also $<g\left(\frac{1}{2} x\right)^{2}\|\rho\|_{p^{\prime}}^{p^{\prime} / \beta}$ for $\|x\| \geqslant 2 c$ so that

$$
\int d x d y\left|K_{1}(x, y)\right|^{2} \rho(y)<\|\rho\|_{p}, c(\operatorname{supp} \rho, t)
$$

and the proposition is proved.

We end this section with the proof of Proposition 10.4:

(i) Let $h_{0}$ be given by equation (2.3) and suppose $h_{0}$ is $C^{\infty}$ in a neighborhood of the closed ball $B$ centered at the origin. Choose $g_{0} \in \delta\left(\mathbf{R}^{N}\right)$ with $g_{0}(0)=1, g_{0}(x)=g_{0}(-x)>0$, supp $\hat{g}_{0} \subseteq B$ and let $g_{\alpha}(x)=g_{0}(\alpha x)$. Define $q_{0}=h_{0}-\sum_{i, j} C_{i j} p_{i} p_{j}$ and suppose $m$ is a positive integer. Then by equation (2.3)

$$
\int\left((-\Delta)^{m} \hat{g}_{\alpha}\right)(p) q_{0}(p) d p=c \int_{|\lambda|>0}|\lambda|^{2(m-1)}\left(1+\lambda^{2}\right) g_{\alpha}(\lambda) d \mu(\lambda) .
$$


If $\alpha \in[0,1]$, supp $\hat{g}_{\alpha} \subseteq B$ so that an integration by parts gives

$$
\left.\left|\int_{|\lambda|>0}\right| \lambda\right|^{2(m-1)}\left(1+\lambda^{2}\right) g_{\alpha}(\lambda) d \mu(\lambda) \mid<c_{m}\left\|\hat{g}_{\alpha}\right\|_{1}=c_{m}^{\prime} .
$$

Using Fatou's lemma and the fact that $\lim _{\alpha \downarrow 0} g_{\alpha}(\lambda)=1$ if $\lambda \neq 0$, we find

$$
\int d \mu(\lambda)\left(1+\lambda^{2}\right)^{m}<\infty \text { for each } m>0 \text {. }
$$

Thus again by equation (2.3), $h_{0} \in C^{\infty}\left(\mathbf{R}^{n}\right)$. By the proof of Lemma 2.7, $\left|\left(\partial_{i} h_{0}\right)(p)\right|^{2}<c^{\prime} h_{0}(p)$, and by (10.4) and (2.3), $\left\|D^{(n)} h_{0}\right\|_{\infty}<\infty$ for all $|n|>1$ so that integration by parts shows

$$
\left(1+x^{2}\right)^{m} K_{t}^{0}(x)<c \int\left|(1-\Delta)^{m} e^{-t h_{0}(p)}\right| d p<\gamma_{m} .
$$

(ii) If the convolution kernel of $e^{-t H_{i}}$ is $j_{i}, i=1,2$, then $K_{t}^{0}(x)=\int j_{1}(x-$ $y) j_{2}(y) d y$. If $j_{1}<g_{i}, g_{i} \in L^{2}$ and $g_{i}$ is decreasing with respect to $\|\cdot\|$, then

$$
\begin{aligned}
K_{t}^{0}(x)< & \int_{\|y\|<\|x\| / 2} g_{1}(x-y) j_{2}(y) d y \\
& \quad+\int_{\|y\|>\|x\| / 2} j_{1}(x-y) g_{2}(y) d y \\
& <g_{1}\left(\frac{1}{2} x\right)+g_{2}\left(\frac{1}{2} x\right) .
\end{aligned}
$$

(iii) From the theory of completely monotone functions [11],

$$
e^{-t g \cdot h_{1}}=e^{-t h_{0}}=\int_{0}^{\infty} d \nu_{t}(\lambda) e^{-\lambda h_{1}}
$$

for some probability measure $\nu_{t}$. Thus $K_{t}^{0}(x)$ is a weighted average of decreasing functions and therefore itself decreasing.

This completes the proof.

Acknowledgements. One of us (I.H.) would like to thank Lon Rosen for a helpful conversation.

\section{BIBLIOGRAPHY}

1. Ju. M. Berezanskii, Expansions in eigenfunctions of selfadjoint operators, Transl. Math. Monographs, Vol. 17, Amer. Math. Soc., Providence, R. I., 1968.

2. A. Beurling and J. Deny, Espaces des Dirichlet I. Le cas elementaire, Acta Math. 99 (1958), 203-224.

3. F. E. Browder, The eigenfunction expansion theorem for the general self-adjoint singular elliptic partial differential operator I. The analytical foundations, Proc. Nat. Acad. Sci. U.S.A. 40 (1954), 454-459.

4. T. Carleman, Sur la théorie mathematique de l'équation de Schrödinger, Ark. Mat. Astronom. Fys. 24B (1934), 1-7.

5. K. M. Case, Singular potentials, Phys. Rev. 80 (1950), 797-806. 
6. M. Combescure and J. Ginibre, Spectral and scattering theory for the Schrödinger operator with strongly oscillating potentials, Orsay (preprint).

7. P. Deift and B. Simon, On the decoupling of finite singularities from the question of asymptotic completeness in two body quantum systems, J. Functional Analysis 23 (1976), 218-238.

8. P. A. M. Dirac, The principles of quantum mechanics, 3rd ed., Oxford Univ. Press, New York, 1947.

9. W. G. Faris, Perturbations and non-normalizable eigenvectors, Helv. Phys. Acta 44 (1971), 930-936.

10. , Self-adjoint operators, Springer-Verlag, Berlin, 1975.

11. W. Feller, An introduction to probability theory and its applications, Vol. II, Wiley, New York, 1971.

12. M. Fukushima, On the generation of Markov processes by symmetric forms, Proc. Second Japan-USSR Sympos. on Probability Theory, Springer-Verlag, Berlin, 1973.

13. I. M. Gel'fand and N. Ya. Vilenkin, Generalized functions, Vol. 4, Academic Press, New York, 1964.

14. B. V. Gnedenko, The theory of probability, Chelsea, New York, 1963.

15. I. Herbst, Spectral theory of the operator $\sqrt{p^{2}+m^{2}}-Z e^{2} / r$, Comm. Math. Phys. 53 (1977), 285-294.

16. B. Simon and R. Höegh-Krohn, Hypercontractive semigroups and two dimensional self-coupled Bose fields, J. Functional Analysis 9 (1972), 121-179.

17. J. Howland, A perturbation-theoretic approach to eigenfunction expansions, J. Functional Analysis 2 (1968), 1-23.

18. T. Ikebe, Eigenfunction expansions associated with Schroedinger operators and their applications to scattering theory, Arch. Rational Mech. Anal. 5 (1966), 1-34.

19. T. Kato, Fundamental properties of Hamiltonian operators of Schrödinger type, Trans. Amer. Math. Soc. 70 (1951), 195-211.

20. ___ Perturbation theory for linear operators, Springer-Verlag, New York, 1966.

21.

22. T. Kato and S. T. Kuroda, Theory of simple scattering and eigenfunction expansions, Functional Analysis and Related Fields, F. E. Browder, editor, Springer-Verlag, New York, 1970.

23. T. Kato, On the eigenfunctions of many-particle systems in quantum mechanics, Comm. Pure Appl. Math. 10 (1957), 151-177.

24. L. D. Landau and E. M. Lifshitz, Quantum mechanics, 2nd ed., Addison-Wesley, Reading, Mass., 1965.

25. F. I. Mautner, On eigenfunction expansions, Proc. Nat. Acad. Sci. U.S.A. 30 (1953), 49-53.

26. E. Nelson, Feynman integrals and the Schrödinger equation, J. Mathematical Phys. 5 (1964), 332-343.

27. G. Nenciu, Eigenfunction expansions for Schrödinger and Dirac operators with singular potentials, Comm. Math. Phys. 42 (1975), 221-229.

28. J. von Neumann, Allgemeine Eigenwertheorie Hermitescher Funktionaloperatoren, Math. Ann. 102 (1929/30), 49-131.

29. M. Reed and B. Simon, Methods of modern mathematical physics. II: Fourier analysis, self-adjointness, Academic Press, New York, 1975.

30. M. Schecter, Hamiltonians for singular potentials, Indiana Math. J. 22 (1972), 483-502.

31. B. Simon, Essential self-adjointness of Schrödinger operators with positive potentials, Math. Ann. 201 (1973), 211-220.

32. __ Essential self-adjointness of Schrödinger operators with singular potentials, Arch. Rational Mech. Anal. 52 (1973), 44-48.

33.

Proc. Amer. Math. Soc. 42 (1974), 395-401.

34. E. M. Stein, Singular integrals and differentiability properties of functions, Princeton Univ. Press, Princeton, N. J., 1970. 
35. D. W. Thoe, Eigenfunction expansions associated with Schrödinger operators in $R_{n}, n>4$, Arch. Rational Mech. Anal. 26 (1967), 335-356.

36. H. F. Trotter, Approximation of semi-groups of operators, Pacific J. Math. 8 (1958), 887-919.

37. F. Treves, Topological vector spaces, distributions and kernels, Academic Press, New York, 1967.

Departument of Physics, Prnnceton University, Princeton, New Jersey 08540

School of Mathematics, Georou Institute of Technolooy, Atranta, Georou 30332 (Current address of A. D. Sloan)

Current address (I. W. Herbst): Department of Mathematics, University of Virginia, Charlottesville, Virginia 22903 